\title{
A NEAR-INFRARED VARIABILITY STUDY OF THE GALACTIC BLACK HOLE: A RED NOISE SOURCE WITH NO DETECTED PERIODICITY
}

\author{
T. Do ${ }^{1}$, A. M. Ghez ${ }^{1}$, M. R. Morris ${ }^{1}$, S. Yelda ${ }^{1}$, L. Meyer ${ }^{1}$, J. R. Lu ${ }^{1}$, S. D. Hornstein ${ }^{2}$, And K. Matthews ${ }^{3}$ \\ ${ }^{1}$ Physics and Astronomy Department, University of California, Los Angeles, CA 90095-1547, USA; tdo@ astro.ucla.edu \\ ${ }^{2}$ Center for Astrophysics and Space Astronomy, Department of Astrophysical and Planetary Sciences, University of Colorado, Boulder, CO 80309, USA \\ ${ }^{3}$ California Institute of Technology, Pasadena, CA, USA \\ Received 2008 May 31; accepted 2008 October 2; published 2009 January 28
}

\begin{abstract}
We present the results of near-infrared (NIR; 2 and $3 \mu \mathrm{m}$ ) monitoring of Sgr A*-IR with 1 minute time sampling using the natural and laser guide star adaptive optics system at the Keck II telescope. Sgr A*-IR was observed continuously for up to $3 \mathrm{hr}$ on each of seven nights, between 2005 July and 2007 August. Sgr A*-IR is detected at all times and is continuously variable, with a median observed $2 \mu \mathrm{m}$ flux density of $0.192 \mathrm{mJy}$, corresponding to $16.3 \mathrm{mag}$ at $K^{\prime}$. These observations allow us to investigate Nyquist sampled periods ranging from about 2 minutes to $1 \mathrm{hr}$. Using Monte Carlo simulations, we find that the variability of Sgr A* in this data set is consistent with models based on correlated noise with power spectra having frequency-dependent power-law slopes between 2.0 and 3.0, consistent with those reported for active galactic nucleus light curves. Of particular interest are periods of $\sim 20$ minutes, corresponding to a quasiperiodic signal claimed based upon previous NIR observations and interpreted as the orbit of a "hot spot" at or near the last stable orbit of a spinning black hole. We find no significant periodicity at any timescale probed in these new observations for periodic signals. This study is sensitive to periodic signals with amplitudes greater than $20 \%$ of the maximum amplitude of the underlying red noise component for light curves with a duration greater than $\sim 2 \mathrm{hr}$ at a $98 \%$ confidence limit.
\end{abstract}

Key words: black hole physics - Galaxy: center - techniques: high angular resolution

Online-only material: color figures

\section{INTRODUCTION}

The existence of a supermassive black hole with a mass of $\sim 4 \times 10^{6} M_{\odot}$ at the center of the Galaxy has now been firmly established from monitoring the orbits of the stars in the nearinfrared (NIR) within 1 arcsec of the location of the associated radio source Sgr A* (e.g., Schödel et al. 2002, 2003; Ghez et al. 2003, 2005a, 2008). Multiwavelength detections of the radio point source at submillimeter, X-ray, and IR wavelengths have also been made, showing that the luminosity associated with the black hole is many orders of magnitude below that of active galactic nuclei (AGNs) with comparable masses (Melia \& Falcke 2001). These observations have also shown that Sgr A* is a variable source (e.g., Baganoff et al. 2003; Mauerhan et al. 2005; Eisenhauer et al. 2005; Hornstein et al. 2007; Marrone et al. 2008; Eckart et al. 2008). Although it is now easily detected in its bright states when its flux increases by up to an order of magnitude over timescales of 1 to $3 \mathrm{hr}$, Sgr A* is difficult to detect in its faintest states at X-ray wavelengths because of the strong diffuse background, and in the NIR because of confusion with nearby stellar sources (Baganoff et al. 2003; Hornstein et al. 2007). Advances in adaptive optics (AO) technology have offered improved sensitivity to IR emission from the location of Sgr A* against the stellar background, such that observations in its faint states are now possible (Ghez et al. 2005b). Hereafter, the IR-luminous source Sgr A*-IR will be referred to simply as Sgr A*, recognizing that it is likely to be coincident with the radio source of that name.

At both NIR and X-ray wavelengths, a possible quasiperiodic oscillation (QPO) signal with $\mathrm{a} \sim 20$ minute period has been reported in light curves of Sgr A* (Genzel et al. 2003; Aschenbach et al. 2004; Eckart et al. 2006). Models that aim to produce QPO signals include both a class of models involving the Keplerian orbits of "hot spots" of plasma at the last stable orbit (Meyer et al. 2006; Trippe et al. 2007) as well as models with rotational modulations of instabilities in the accretion flow (Falanga et al. 2007). Since the orbital period at the last stable orbit of a nonspinning black hole is $32\left(M_{\mathrm{bh}} / 4.2 \times 10^{6} M_{\odot}\right)$ minutes, this putative periodic signal has been interpreted as evidence for a spinning black hole. The challenges for these claims are the relatively short time baselines of the observations (only a few times the claimed period), the low amplitude of the possible QPO activity, and the level of rigorous assessment of the statistical significance of the claimed periodicity.

An alternative explanation for peaks in the periodograms seen in previous studies and interpreted as a periodic signal is that they are a sign of a frequency-dependent physical process, commonly known as red noise (Press 1978). The power spectrum of such a physical process will display an inverse power-law dependence on frequency, which manifests as light curves with large amplitude variations over long timescales and small amplitude variations over short timescales. The power spectrum of any individual realization of a red noise light curve will show statistical fluctuations around the intrinsic power-law function, creating spurious peaks that can lead to an interpretation of periodic activity. Variability studies of other accreting black hole systems such as AGNs and Galactic X-ray binaries have shown that their power-spectral densities (PSDs) are consistent with red noise. Several physical models have been proposed to produce the red noise light curves seen in AGNs (e.g., Lyubarskii 1997; Armitage \& Reynolds 2003; Vaughan et al. 2003); one common model that results in a red noise spectrum is from fluctuations in the physical parameters, such as the gas densities and accretion rate, at different radii of a turbulent magnetohydrodynamic accretion disk (Kataoka et al. 2001). While QPO signals have been unambiguously confirmed 
Table 1

Summary of Observations of Sgr A*

\begin{tabular}{|c|c|c|c|c|c|c|c|c|c|c|c|c|c|}
\hline $\begin{array}{l}\text { Date } \\
\text { (UT) }\end{array}$ & Filter & $\begin{array}{c}\text { Start Time } \\
\text { (UT) }\end{array}$ & $\begin{array}{c}\text { End Time } \\
\text { UT }\end{array}$ & $\mathrm{N}_{\mathrm{obs}}$ & $\begin{array}{c}\text { Median Samp. } \\
\text { (s) }\end{array}$ & $\begin{array}{l}\text { Duration } \\
\text { (minute) }\end{array}$ & Dithered $^{\mathrm{a}}$ & $\begin{array}{c}\text { Strehl }^{b} \\
(\%)\end{array}$ & $\begin{array}{c}\text { FHWM } \\
\text { (mas) }\end{array}$ & $\begin{array}{c}\text { Mean Flux } \\
(\mathrm{mJy})\end{array}$ & $\begin{array}{c}\sigma^{\mathrm{b}} \\
(\mathrm{mJy})\end{array}$ & $\begin{array}{c}\text { S0-17 }{ }_{\text {phot }}{ }^{\mathrm{d}} \\
(\%)\end{array}$ & $\begin{array}{c}\mathrm{S} 2-133_{\text {phot }}{ }^{\mathrm{d}} \\
(\%)\end{array}$ \\
\hline $2005 \mathrm{Jul} 28^{\mathrm{e}}$ & $L^{\prime}$ & $06: 10: 52$ & $09: 09: 23$ & 144 & 69 & 180 & No & 70 & 81 & 4.74 & 1.7 & $\ldots$ & $\ldots$ \\
\hline 2006 May 3 & $K^{\prime}$ & $10: 54: 30$ & $13: 14: 12$ & 116 & 51 & 140 & Yes & 34 & 60 & 0.29 & 0.15 & 7 & 13 \\
\hline 2006 Jun 20 & $K^{\prime}$ & $08: 59: 22$ & $11: 17: 54$ & 70 & 51 & 79 & Yes & 24 & 71 & 0.24 & 0.18 & 7 & 22 \\
\hline 2006 Jun 21 & $K^{\prime}$ & $08: 52: 26$ & $11: 36: 53$ & 164 & 51 & 164 & Yes & 33 & 61 & 0.19 & 0.07 & 4 & 17 \\
\hline $2006 \mathrm{Jul} 17^{\mathrm{f}}$ & $K^{\prime}$ & $06: 45: 29$ & 09:54:03 & 70 & 147 & 189 & No & 35 & 59 & 0.15 & 0.05 & 5 & 22 \\
\hline 2007 May 18 & $K^{\prime}$ & $11: 34: 10$ & $13: 52: 39$ & 77 & 51 & 84 & Yes & 36 & 59 & 0.29 & 0.14 & 7 & 10 \\
\hline 2007 Aug 12 & $K^{\prime}$ & $06: 67: 10$ & $07: 44: 38$ & 62 & 51 & 57 & Yes & 33 & 58 & 0.16 & 0.02 & 4 & 7 \\
\hline
\end{tabular}

Notes.

${ }^{a}$ When the observations are dithered, the time interval between dithers is 3 minutes.

b Average for the night. Strehl ratios and FWHM measurements made on IRS 33N for all $K^{\prime}$ data and IRS $16 \mathrm{C}$ for $\mathrm{L}^{\prime}$ data.

${ }^{\mathrm{c}}$ Flux values are observed fluxes, not dereddened.

d Photometric precision for the two comparison sources S0-17 and S2-133, with mean fluxes 0.38 mJy and $0.15 \mathrm{mJy}$ respectively.

e NGS mode.

${ }^{f}$ Previously reported in Hornstein et al. (2007).

in X-ray binaries, no QPO signals in AGNs have been shown to be statistically different than red noise (Benlloch et al. 2001; Vaughan 2005). Recent work by Bélanger et al. (2009) have also shown that a statistical analysis of X-ray light curves of Sgr $\mathrm{A}^{*}$, when including the contribution from red noise, show no indications of a QPO signal.

High sensitivity and high angular resolution NIR observations of Sgr A* have been obtained at the Keck II telescope utilizing new improvements in adaptive optics technologies to investigate the existence of a QPO signal as well as the timing properties of $\mathrm{Sgr} \mathrm{A}^{*}$. These observations are described in Section 2. In order to test whether the variability of $\mathrm{Sgr} \mathrm{A}^{*}$ has the characteristics of red noise and to examine the possibility of a periodic signal, we have carried out a statistical analysis of the timing properties of the observed light curves that includes the possible contribution of red noise in the power spectrum to establish the significance of peaks in the periodograms. We find that the NIR variability of Sgr A* is entirely consistent with red noise, with no periodic signals detected on any night. Sections 3.2.1 details our analysis of the light curves. Lastly, in Section 4, we discuss the implications of our results for Keplerian models of the Sgr A* flux variability and compare the timing properties of Sgr A* with those of AGNs.

\section{OBSERVATIONS AND DATA REDUCTION}

The Galactic center has been extensively imaged between 2005 and 2007 with the Keck II 10 m telescope using the natural and laser guide star adaptive optics (NGS and LGS AO; Wizinowich et al. 2006; van Dam et al. 2006) system and the NIRC2 NIR camera (P.I. K. Matthews). For this study we include all nights of LGS-AO observations at $K^{\prime}(2 \mu \mathrm{m})$ that had sampling of 1-3 minutes, a total time baseline of at least $\sim 1 \mathrm{hr}$, and at least 60 data points. We also include one night of NGS observations at $L^{\prime}$ that satisfied the same criteria. As summarized in Table 1, this resulted in a selection of seven data sets with durations ranging from 80 minutes to $3 \mathrm{hr}$.

A detailed description of LGS AO observations of the Galactic center is described in Ghez et al. (2005b); here, we only summarize the setup for our observations. The laser guide star was propagated at the center of our field and for low-order tip-tilt corrections, we used the $R=13.7$ mag star, USNO $0600-28577051$, which is located $\sim 19^{\prime \prime}$ from Sgr A*. Most of the images were obtained using the $K^{\prime}$ band-pass filter $\left(\lambda_{o}=\right.$ $2.12 \mu \mathrm{m}, \Delta \lambda=0.3 \mu \mathrm{m})$ and were composed of ten coadded $2.8 \mathrm{~s}$ exposures, for a total integration time of $28 \mathrm{~s}$. The remaining set of observations from 2005 July 28 was taken through the $L^{\prime}$ band-pass filter $\left(\lambda_{o}=3.78 \mu \mathrm{m}, \Delta \lambda=0.7 \mu \mathrm{m}\right)$. For five of the $K^{\prime}$ nights, the time interval between each image is about $50 \mathrm{~s}$, with dithers every 3 minutes. $K^{\prime}$ images from 2006 July 17 were sampled at 3 minute intervals but were not dithered. The $L^{\prime}$ observations had 1 minute sampling, and were also not dithered. The 3 minute dithers affect the timing analysis by the presence of a spike at that frequency in the periodograms (this is well reproduced by Monte Carlo (MC) simulations of the effects of sampling).

Photometry was performed on the individual images using the point spread function (PSF) fitting program StarFinder (Diolaiti et al. 2000). The program was enhanced as described in Hornstein et al. (2007) to include the a priori knowledge of the location of the NIR position of Sgr A* and nearby sources in order to facilitate the detection of Sgr A* at faint flux levels in these short exposures. To do this, for each night of observation, the position of Sgr A* and nearby sources was determined in a nightly averaged image produced by a weighted average of individual images from that night (see Figure 1). We then used the knowledge of the location of all the sources as fixed inputs into StarFinder to more accurately fit for the flux contribution of sources near Sgr A* in the individual short exposure images. To compensate for seeing changes through the night, a different PSF was constructed for each image. We also include only images with Strehl ratios greater than $20 \%$ to minimize large errors in the photometry from bad seeing conditions, which resulted in dropping only about ten data points out of all nights. On average, the Strehl ratio at $K^{\prime}$ was $32 \%$, with the full width of the core at half-maximum intensity (FWHM) $\sim 60$ mas as measured from the relatively isolated star IRS $33 \mathrm{~N}$. We are able to detect $\operatorname{Sgr} \mathrm{A}^{*}$ at all times, even at its faintest flux levels. The gaps in the data are from technical disruptions in the observations.

Photometric calibrations were performed relative to the list of nonvariable sources from Rafelski et al. (2007) at $K^{\prime}$ and IRS $16 \mathrm{C}\left(L^{\prime}=8.14 \mathrm{mag}\right)$ and $\mathrm{IRS} 16 \mathrm{NW}\left(L^{\prime}=8.43 \mathrm{mag}\right)$ at $L^{\prime}$ (Blum et al. 1996). The photometric error at each flux density level seen in Sgr A* was estimated by fitting a power law to the rms uncertainty in the flux for all nonvariable stars in the 

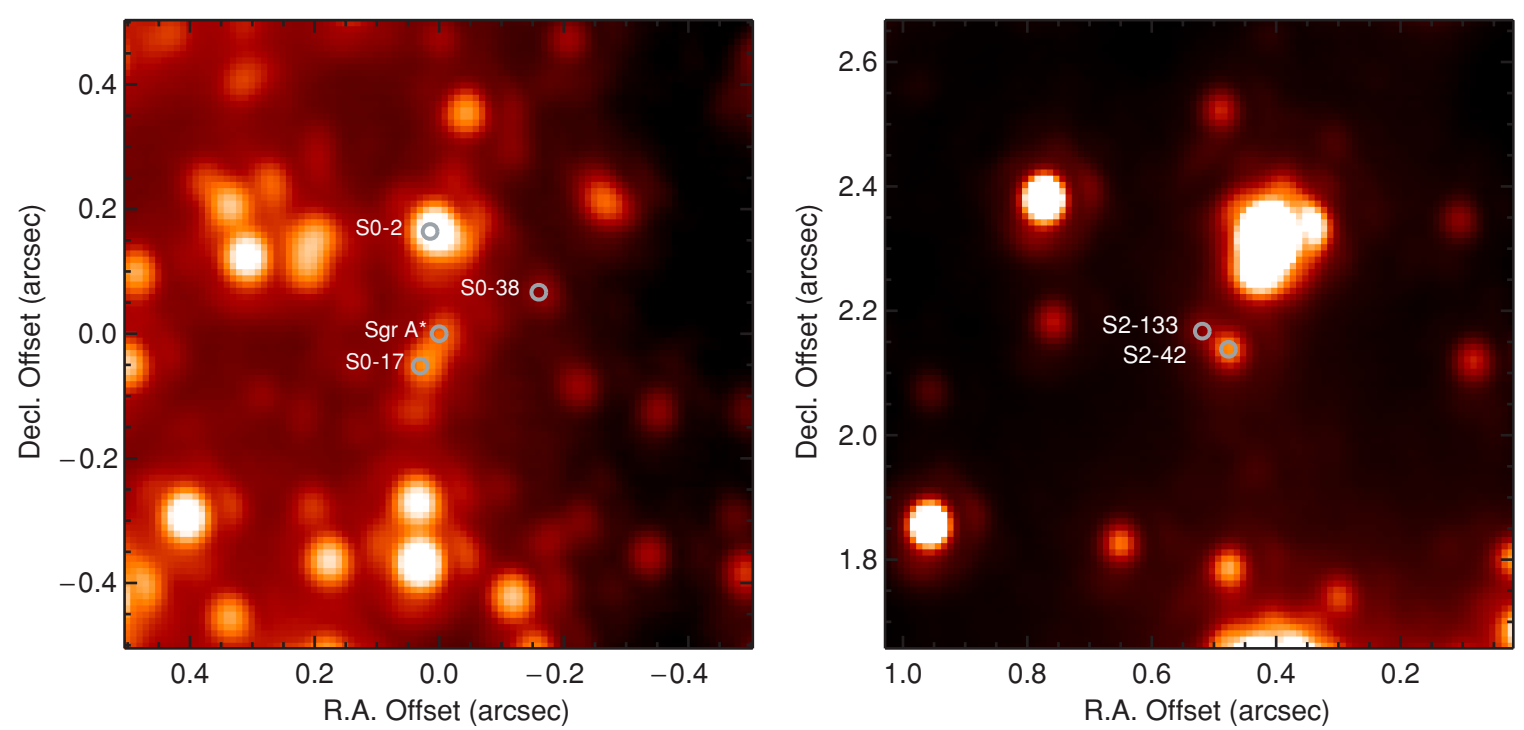

Figure 1. Left: $K^{\prime}$ image from 2006 May 3 of the central 0.5 around Sgr A* with a logarithmic intensity scale so that the faint sources can be more easily seen Sgr A* $\left(K^{\prime}=15.8\right.$ in this image) is in the center of the image along with the comparison stars, S0-2, S0-17, and S0-38. The image is oriented with north up and east to the left, with offsets in the projected distance from Sgr A*. Right: Image from the same night of the pair of comparison sources, S2-42 $\left(K^{\prime}=15.5\right)$ and S2-133 $\left(K^{\prime}=16.7\right)$, with a flux ratio similar to that of S0-17 and Sgr A* when Sgr A* is faint. These two stars also have a similar separation in the plane of the sky as S0-17 and $\mathrm{Sgr} \mathrm{A}^{*}(\sim 50 \mathrm{mas})$.

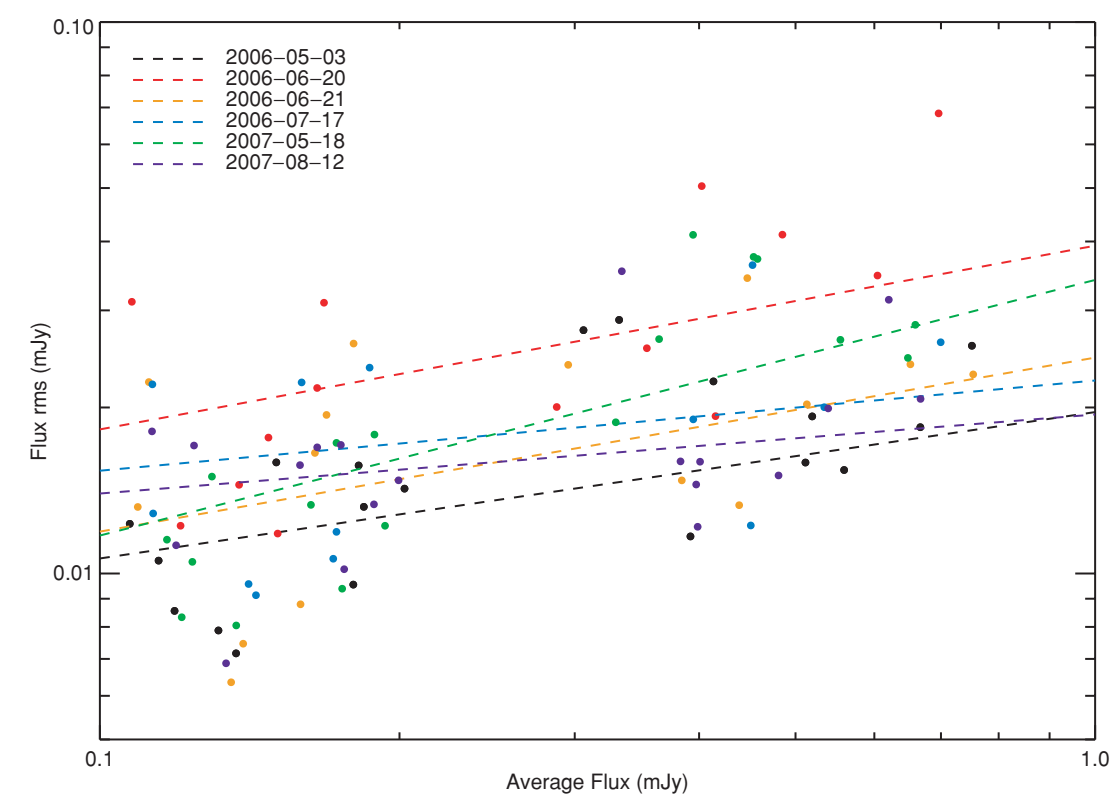

Figure 2. Plot of power-law fits to the rms fluxes for stars within 0.5 of Sgr A* with brightnesses within the range of observed brightness variations in the $K^{\prime}$ light curves from Sgr A*. Photometric noise properties are similar for each night.

same range of brightnesses observed for Sgr A* within 0".5 of the black hole (see Figure 2). We find the typical dependence of the photometric error, $\sigma$, on the flux density, $F$, to be $\sigma \approx$ $0.2 F^{0.3} \mathrm{mJy}$. The flux measurement uncertainties are comparable for all nights except 2006 June 20, the night with the worst seeing. Within the range of observed Sgr A* fluxes, we are on average able to achieve between $3 \%$ and $15 \%$ relative photometric precision for each $28 \mathrm{~s} K^{\prime}$ exposure. A source of systematic error in the flux measurements is the proximity of Sgr $\mathrm{A}^{*}$ to unresolved sources, which could contribute to the flux. This contribution is only likely to have an impact when Sgr A* is faint (Hornstein et al. 2007), but for the purpose of this variability study, this effect is likely only a systematic offset in the mean flux density and a source of white noise (for more details, see Section 3.1). For comparison to Sgr A*, we also analyze the light curves of the nearby stars $\mathrm{S} 0-17$ and $\mathrm{S} 0-38$ at $K^{\prime}$ and $\mathrm{S} 0-2$ at $L^{\prime}$. S0-17 ( $\left.K^{\prime}=15.5 \mathrm{mag}\right)$ was chosen because it is spatially closest to $\mathrm{Sgr} \mathrm{A}^{*}$, with a projected distance from Sgr A* of $\sim 56$ mas in 2006 May to $\sim 48$ mas in 2007 August; monitoring S0-17 is helpful to ensure that the variations in flux seen in Sgr A* are not a systematic effect of seeing or bias from nearby sources. The star S0-38 $\left(\sim 0.11 \mathrm{mJy}, K^{\prime} \sim 17 \mathrm{mag}\right), \sim 0^{\prime \prime} 2$ from Sgr A*, was chosen as a stellar reference because it has a similar flux to the faintest observed emission and given its proximity to Sgr A*, its photometry will be affected similarly from the unresolved stellar background. Figure 1 shows an image of this region and the location of the comparison sources with respect to Sgr A*. 

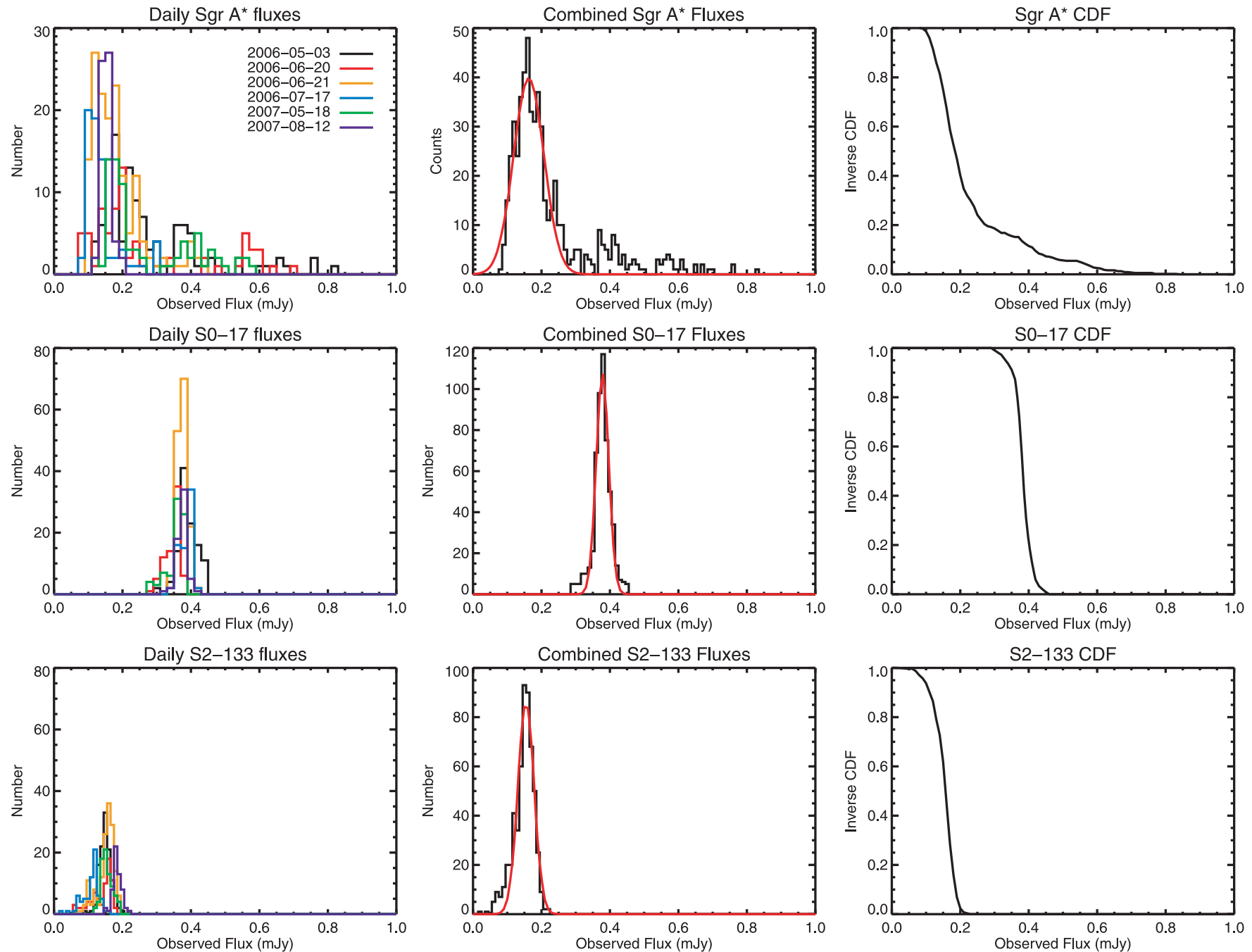

Figure 3. Top: Sgr A* flux distribution for each of the $6 K^{\prime}$ nights (left), the combined fluxes from all nights (middle), and the inverse CFD for the combined fluxes (right). Middle: Corresponding plots for S0-17, the nonvariable comparison source used in this study. The total histogram of fluxes observed from S0-17 is consistent with a single Gaussian. Bottom: Flux distribution for S2-133, a $K^{\prime}=16.7$ mag star for comparison with the faint states of Sgr A*.

In order to characterize possible effects on the photometry of Sgr A* by S0-17, we also use the photometry of two stars with separations and flux ratios similar to that of S0-17 and Sgr A* when Sgr A* is faint. The two stars, S2-42 $\left(K^{\prime}=15.5\right)$ and S2-133 $\left(K^{\prime}=16.7\right)$, are located about $2^{\prime \prime}$ from Sgr A* and are separated by $\sim 50$ mas (Figure 1). The rms variability of S2-42 and S1-133 is about $5 \%$ and $15 \%$, respectively, similar to the photometric precision we would have predicted based upon our power-law fits to the rms stability of stars near Sgr A*. Thus, we can be confident that the photometry of Sgr A* at its faintest is similar to stars of that magnitude despite the proximity of S0-17.

\section{RESULTS AND ANALYSIS}

\subsection{Flux Distribution}

In order to characterize the range of fluxes observed from Sgr A*, we have constructed histograms of fluxes from each night as well as the combined histogram from all nights (Figure 3). Unless otherwise stated, the fluxes in this paper are observed fluxes and not corrected for extinction to Sgr A*. Where indicated, dereddened fluxes have been calculated by assuming $A_{v}=30$ (Moneti et al. 2001) and extinction law $A_{K^{\prime}}=0.1108 A_{v}$ (Rieke \& Lebofsky 1985). The comparison sources do not appear to be variable on the timescales probed in this study and have fluctuations consistent with Poisson noise. The flux distribution for the star S0-17 is consistent with a Gaussian centered at $0.37 \mathrm{mJy}$ at $K^{\prime}$, with a standard deviation of $0.02 \mathrm{mJy}$; this suggests that we are able to reproduce the flux of S0-17 at the 5\% level between different nights. A bias in the photometry from the proximity of S0-17 to the light curve from Sgr A* should manifest itself as a difference in the mean flux of S0-17 between 2006 and 2007 because S0-17 moved closer to Sgr A* in the plane of the sky between the two years. The fact that we observe the same mean flux from S0-17 between different years is also confirmation that there is little bias in the photometry of either S0-17 or Sgr A*. The light curves of S0-17 are also stable on each night, independent of the flux of Sgr A* and shows no greater variance than other stars of the same brightness in the region (within 0'.5 of Sgr A*), showing that PSF fitting from StarFinder is able to properly account for the flux of both sources.

The cumulative distribution function (CFD) for Sgr A* shows that it is brighter than S0-17 about $15 \%$ of the time at $K^{\prime}$. The median flux density of Sgr A* is at $0.192 \mathrm{mJy}\left(K^{\prime}=16.3 \mathrm{mag}\right)$, or a dereddened flux of $4.10 \mathrm{mJy}$. The flux histogram for Sgr $\mathrm{A}^{*}$ is not well fitted by a Gaussian because it has a long tail in the distribution of flux densities at high-flux densities. However, 

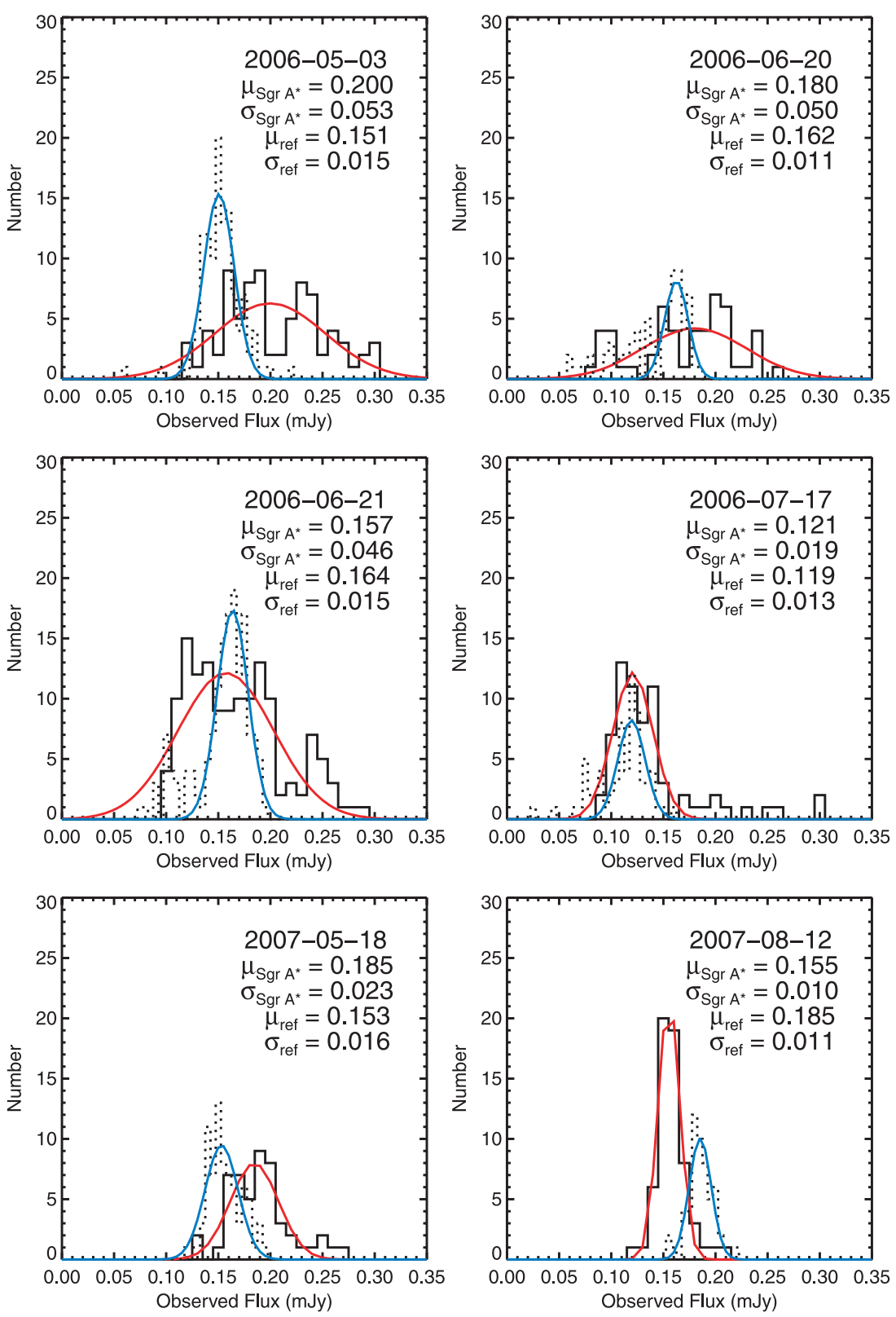

Figure 4. Flux distribution of Sgr A* (solid) of fluxes less than $0.3 \mathrm{mJy}$ and the reference source, S2-133 (dotted) for each night along with the best-fit Gaussian. On four of the six $K^{\prime}$ nights, the width of the Sgr A* flux distribution is greater than the reference, while in the remaining two nights, the widths are comparable. The labels are in units of mJy.

(A color version of this figure is available in the online journal.)

if the tail of the distribution is excluded, the flux distribution below $0.3 \mathrm{mJy}$, is well fitted by a Gaussian with a mean of 0.158 $\mathrm{mJy}$ and a standard deviation of $0.05 \mathrm{mJy}$. The latter is larger than nearby sources with comparable flux densities, indicating that $\mathrm{Sgr} \mathrm{A}^{*}$ is an intrinsically variable source; for example, the flux distribution for S2-133 has an FWHM of $\sim 0.02 \mathrm{mJy}$ (see Figure 4).

The flux density distribution of Sgr A* for the $L^{\prime}$ night shows a much larger width than the comparison star S0-2 (Figure 5). The distribution appears to be symmetric about the mean and the CDF is consistent with a Gaussian with mean flux density of $7.69 \mathrm{mJy}$ with $\sigma=2.81 \mathrm{mJy}$, compared to S0-2 with a mean flux density of $7.67 \mathrm{mJy}$ with $\sigma=0.37 \mathrm{mJy}$.

While the bright flux levels ( $>0.3 \mathrm{mJy}$ ) can be unambiguously attributed to the black hole, the source of emission from the location of Sgr $\mathrm{A}^{*}$ when it is faint is less certain. The region immediately around Sgr A* is also the location of peak stellar density, which raises the possibility of flux contamination from either an unresolved population of stars or a very faint star in a close orbit at that location. We find that the faintest observed flux density at $K^{\prime}$ is $0.082 \pm 0.017 \mathrm{mJy}\left(K^{\prime}=17.2\right)$ or $1.75 \pm 0.36$ mJy dereddened from the location of Sgr A* (consistent with limits observed by Hornstein et al. 2002). Comparison of the flux distribution of S2-133 with that of Sgr A* below $0.3 \mathrm{mJy}$ shows that, for four of the six $K^{\prime}$ nights, Sgr $\mathrm{A}^{*}$ has a larger variance than S2-133 (see Figure 4), indicating that Sgr A* is more variable than expected for a stellar source even at the faintest levels. On the remaining two nights, the variance of Sgr A* is similar to that of S2-133. On one of these two nights, 2007 August 12, Sgr A* was fainter than $0.22 \mathrm{mJy}$ for 

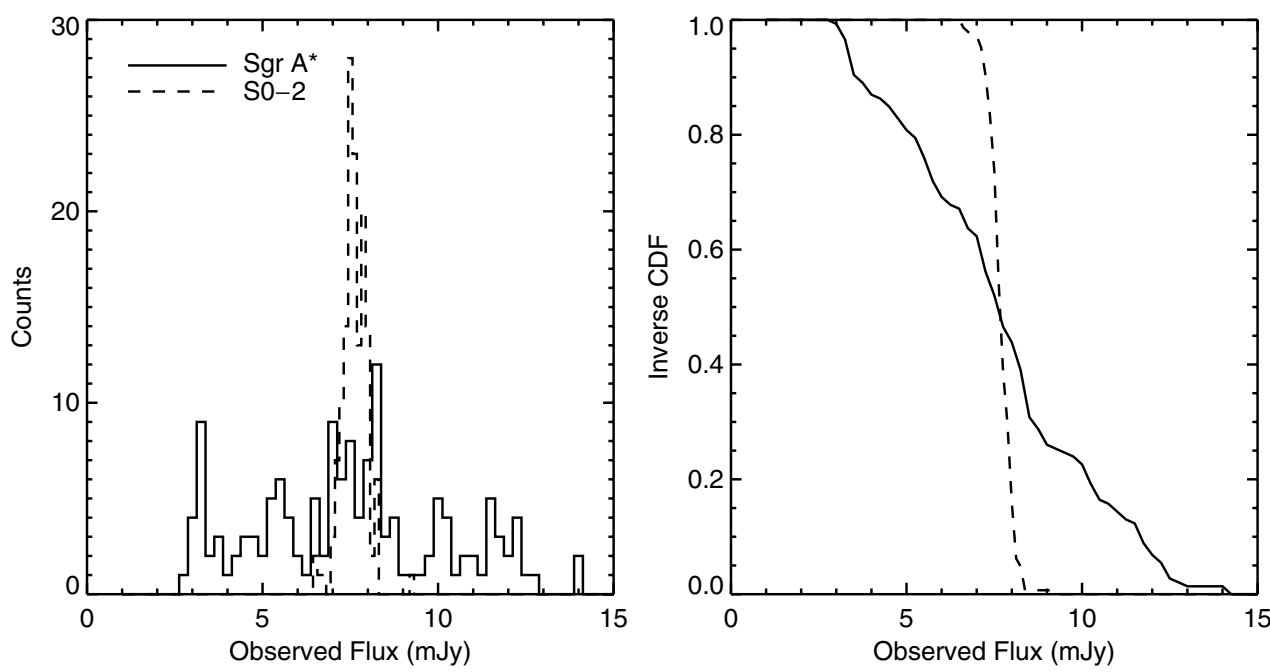

Figure 5. Left: Sgr A* and S0-2 flux distributions on the night of 2005 July 28 at $L^{\prime}$. Right: Corresponding CFD for Sgr A* and S0-2. Both CDFs are consistent with Gaussian distributions.

the entire duration of our observation, which makes this night ideal for timing analysis of Sgr A* at its faintest flux density levels. Though the flux distribution looks similar to a star, the structure function and the periodogram show a slightly steeper slope than expected for Gaussian noise and as compared to the stellar stellar comparison sources (see Sections 3.2.1 and 3.2.2). Furthermore, the $K^{\prime}-L^{\prime}$ color for 2006 July 17, previously reported in Hornstein et al. (2007), is constant and significantly redder, even at its faintest on that night, than from a stellar source. At the faintest fluxes, between 0.10 and $0.15 \mathrm{mJy}$, the mean $K^{\prime}-L^{\prime}$ spectral slope, corrected for extinction, of Sgr A* has an average power-law exponent of $-0.17 \pm 0.32$, compared to a slope of $-0.6 \pm 0.2$ from Hornstein et al. (2007). We estimate that a stellar source, which would have a dereddened spectral slope of 2, could contribute a maximum of about $35 \%$ of the flux to account for the difference in the spectral slope. This leads us to conclude that, even when the emission is faint, a large fraction of the flux arising from the location of Sgr A* is likely nonstellar and can be attributed to physical processes associated with the black hole.

\subsection{Light Curves and Timing Analysis}

Figures 6 and 7 show the resulting light curves for Sgr A* and a nonvariable comparison source for each night of observation. While comparison sources show no significant time variable emission, Sgr A* shows variations on timescales ranging from minutes to hours, with peak emission that can be 10 times higher than during its faintest states. The emission peaks are time symmetric, with similar rise and fall times.

In order to characterize the variability of Sgr $\mathrm{A}^{*}$, we have carried out the following three different approaches to timing analysis: (1) periodograms, (2) structure functions, and (3) autocorrelations. The periodogram analysis, presented in Section 3.2.1, is effective at pulling out periodic structure in light curves and therefore is optimal for assessing the presence of any periodicities, such as the proposed $\sim 20$ minute QPO reported in previous experiments (e.g., Genzel et al. 2003; Eckart et al. 2006). Both the periodogram and the structure function can also be used to measure the underlying PSD, which can then be used to explore similarities to the variability observed in AGNs. We also compute the autocorrelation for each night to look for possible differences in the variability at each timescale between each night.

\subsubsection{Periodogram}

It is important to consider all possible sources of noise when testing for periodicity in light curves. While peaks in the periodograms are a good place to start searching for periodicity, the peaks must have significantly more power than those produced by nonperiodic processes to be unambiguously attributed to a true periodicity in any variable source. White (Gaussian) noise processes are unlikely to lead to large peaks in the periodograms because they contribute equal power at all frequencies. However, time-correlated physical processes can result in variability that is frequency dependent. One common variability characteristic - often seen in AGN light curvesis red noise, which can lead to spurious signals in a power spectrum or periodogram from a data set having a time baseline only a few times longer than that of the putative period, since it will show large amplitude fluctuations at low frequencies and small amplitudes at high frequencies. This can lead to relatively large stochastic peaks in the power spectrum at low frequencies, far above what would be expected from white noise. We emphasize that, although the term for this type of power-law dependence of the flux variability is "red noise," this variability arises from physical processes from the source and is not a result of measurement uncertainties such as Poisson noise, which behaves like white noise in its power spectrum.

One of the goals in this timing analysis is to test whether a purely red noise model can explain the variability of Sgr A*. The PSD of a red noise light curve is a power law, with greater power at lower frequencies: $P(f) \equiv f^{-\alpha}$, where $f$ is the frequency and $\alpha$ is the power-law index. For example, $\alpha=0$ for white noise and $\alpha=1$ for classical flicker noise (Press 1978). All red noise simulations in this paper were produced by an algorithm detailed in Timmer \& Koenig (1995), which randomizes both phase and amplitude of an underlying power-law spectrum and then inverse Fourier transforms it into the time domain to create light curves. Our procedure for producing simulated light curves is as follows: (1) A light curve is produced from a PSD with a specific power-law slope evenly sampled at half the shortest observed time sampling interval, with a duration at least 10 times as long as the observed light curve (rounded up 

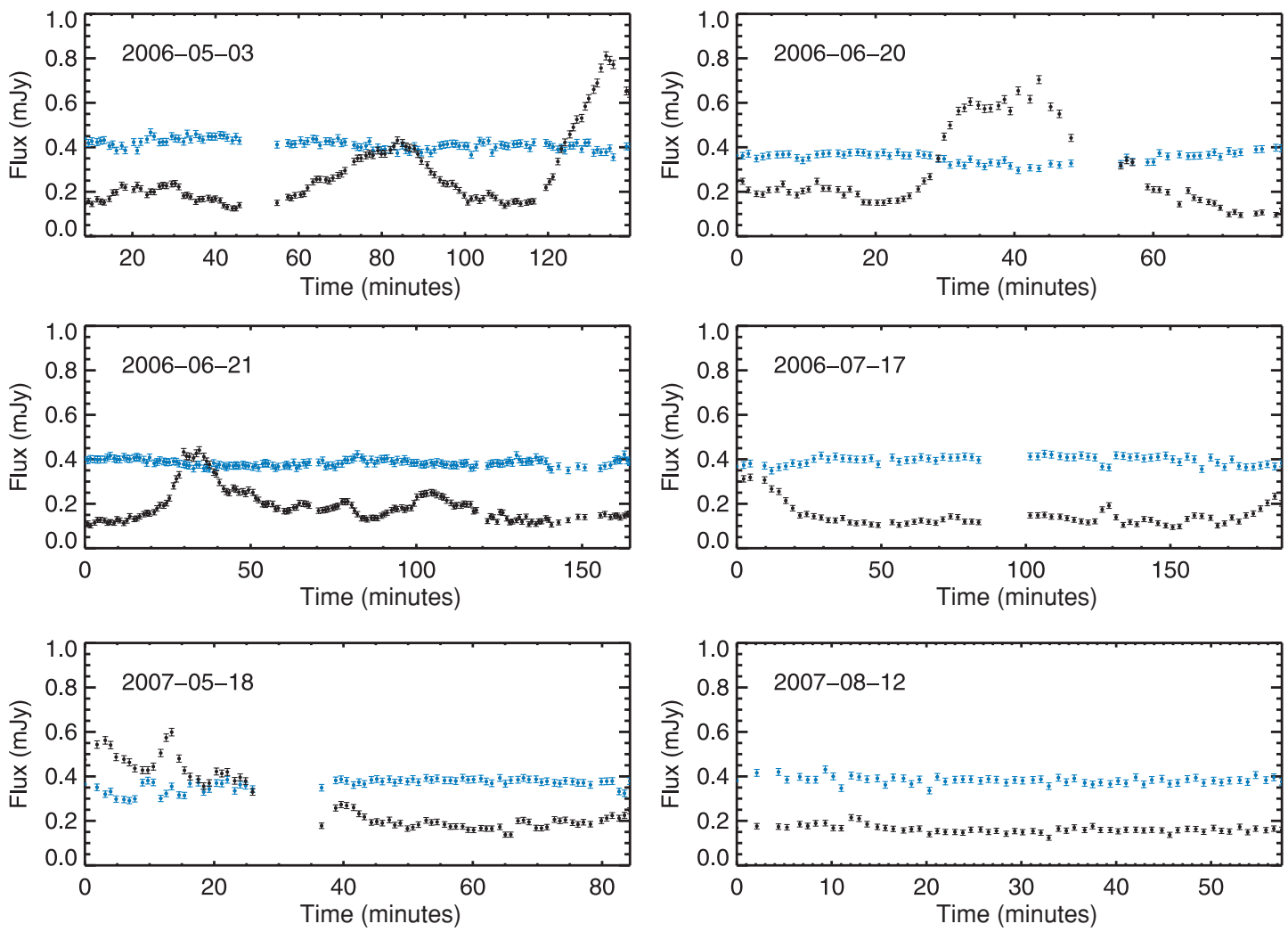

Figure 6. Sgr A* light curves (black) at $K^{\prime}$ with the star S0-17 (blue) for comparison.

(A color version of this figure is available in the online journal.)

to the nearest power of 2 for computational efficiency of the fast Fourier transform). This length was chosen based upon the suggestion by Uttley et al. (2002) to avoid a "red noise leak" where power is distributed from frequencies lower than that sampled by the observation into observed frequencies. We find aliasing to be a negligible effect, because the light curves are generated at higher temporal resolution than the observations. (2) This light curve is then split into 10 nonoverlapping segments to reduce the simulation time (Uttley et al. 2002). Each segment is then resampled at the exact sampling times used during the specific night that we are simulating. (3) Since the simulation has an arbitrary flux scale, we scale the light curves to have the same mean flux level and standard deviation as that night. We also include the effects of measurement noise in the simulations by adding Gaussianly distributed noise to each simulated data point. We use our measurements of the photometric error as a function of flux densities (Section 2) of nonvariable stars within $00^{\prime} .5$ of Sgr A* to account for the flux-density dependence in the noise for each simulated data point.

Instead of computing the PSD, which is often used for evenly sampled data, we searched for periodicity by computing a related function for unevenly sampled data: the normalized Lomb-Scargle periodogram (Press \& Rybicki 1989); given a set of data values $h_{i}, i=1, \ldots, N$ at times $t_{i}$ the periodogram is defined as

$$
\begin{aligned}
P_{N}(\omega) \equiv & \frac{1}{2 \sigma^{2}}\left\{\frac{\left[\sum_{j}\left(h_{j}-\bar{h}\right) \cos \omega\left(t_{j}-\tau\right)\right]^{2}}{\sum_{j} \cos ^{2} \omega\left(t_{j}-\tau\right)}\right. \\
& \left.+\frac{\left[\sum_{j}\left(h_{j}-\bar{h}\right) \sin \omega\left(t_{j}-\tau\right)\right]^{2}}{\sum_{j} \sin ^{2} \omega\left(t_{j}-\tau\right)}\right\},
\end{aligned}
$$

where $\omega$ is the angular search frequency, $\bar{h}$ and $\sigma^{2}$ are the mean and variance of the data, respectively. The constant $\tau$ is an offset introduced to keep the periodogram phase invariant:

$$
\tan (2 \omega \tau)=\frac{\sum_{j} \sin 2 \omega t_{j}}{\sum_{j} \cos 2 \omega t_{j}} .
$$

Since the periodogram is normalized by the variance of the flux, a light curve consisting of only white noise, or equivalently, red noise with a power law $\alpha=0$, will have an average power of 1 at all frequencies.

The normalized Lomb-Scargle periodogram was computed for each light curve, oversampled by a factor of 4 times the independent Fourier intervals in order to increase the sensitivity to periods between the Fourier frequencies (Figures 7 and 8 ). Assuming that the physical source of the variability is stationary, we averaged together the periodograms for the five $K^{\prime}$ nights that have durations longer than 80 minutes (Figure 9). The combined periodogram excludes the 2007 August 12 night because it is less than an hour long, leading to poor sampling at low frequencies compared to the other nights. We combined the periodograms by averaging the Lomb-Scargle power at linearly space frequency bins. The combined periodogram is consistent with red noise, except for the peak corresponding to the timescale of the 3 minute dithers. To characterize the underlying spectrum, we have performed MC simulations combining red noise light curves with the same sampling as the data set. We tested several different underlying PSD and found that the combined periodogram is consistent with power-law indices between 2.0 and 3.0, with no periodic components. This model is able to reproduce the slope of the periodogram, the increase in power at 3 minutes from dithering, and the flattening of the periodogram at very low frequencies caused by poor sampling 

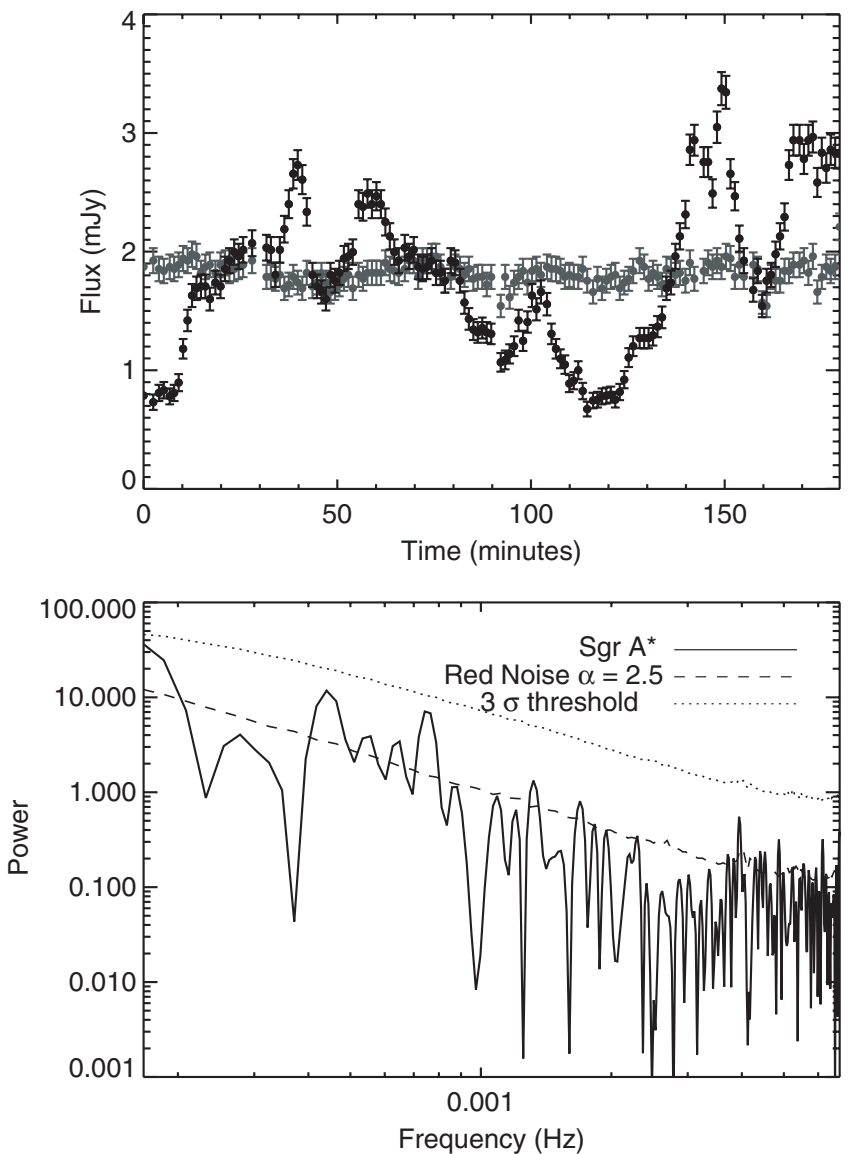

Figure 7. Top: Light curve of Sgr A* (black) and comparison source S0-2 (gray) at $L^{\prime}$ on 2005 July 28 . Bottom: Normalized Lomb-Scargle periodograms of Sgr A* on this night (black). The $3 \sigma$ significance threshold determined from MC simulations of red noise with a power-law index, $\alpha=2.5$ is also plotted (dotted). The average of $10^{5}$ periodograms with $\alpha=2.5$ at the same time sampling is also shown for comparison (dashed line).

at those frequencies. The 3 minute peak in the periodogram is reproduced very well by the simulations, showing that the simulations are correctly accounting for the effects of sampling. Figure 9 shows the results of MC simulations with power-law indices $1.5,2.0,2.5$, and 3.0. The simulations shows that the resulting periodograms tend to be flatter than the intrinsic PSD because the limited time sampling at low frequencies results in poor sensitivity to long timescale variations characteristic of steeper power laws. Because the periodogram sampling is poor at frequencies below 40 minute we will ignore those frequencies in all subsequent analysis. We find the resulting periodograms show less variation for intrinsic PSD $\alpha>2$, which suggests that we have a better constraint on the lower limit than on the upper limit of our estimate for the slope of the PSD. While red noise models with values of $\alpha$ between 2.0 and 3.0 appear to be consistent with the average periodogram, we will use $\alpha=2.5$ for the simulations in this work to provide a baseline for comparison. More light curves will be necessary to determine a reliable intrinsic PSD of Sgr A* (if it does not vary between nights). Where appropriate, we have also run simulations with a range of $\alpha$ values to investigate its effects on the statistical significance.

Although there is no evidence for QPO activity in the combined periodogram, we also test the case of a transient QPO phenomenon in each night. We therefore used our best fit of $\alpha=2.5$ for the power law of the combined periodogram to test each night for statistically significant deviations from a purely red noise model. Our criterion for a statistically significant peak in the periodogram is that its power must be above the 99.7\% $(3 \sigma)$ confidence interval from a MC simulation with $10^{5}$ realizations of red noise light curves. This method of establishing the significance of peaks in the periodogram is similar to the one proposed by Vaughan (2005) for evenly sampled data, but modified here to account for our unevenly sampled data set by using the Lomb-Scargle periodogram instead of the Fourier transform. The resulting periodograms are shown in Figure 8. The individual periodograms show peaks at low frequencies, but these appear to be consistent with red noise, with no peaks having power greater than the $3 \sigma$ threshold derived from the MC simulations. No significant peaks were found when we repeated the same procedure for PSD powerlaw indices between 1.0 and 3.0.

The periodograms of the comparison sources are much flatter than that of $\operatorname{Sgr} \mathrm{A}^{*}$, as expected for white noise sources. However, there is some power at very low frequencies ( $<40$ minutes), which may be the result of some small timecorrelated systematic in the observation such as variations in seeing or AO performance over a night. Light curves and periodograms from 2006 June 21 of the three comparison sources S0-17, S0-38, and S2-133 are shown in Figure 10. The periodograms of these sources from the other nights of observations are also comparably flat.

By adding an artificial sinusoidal periodic signal to the red noise simulations, we can address our sensitivity for detecting QPOs in the presence of red noise. In order to test this, a periodic sinusoidal signal was added to a simulated light curve with a red noise PSD slope of 2.5 , with an amplitude that is a fraction of the maximum signal in the underlying red noise light curve. This fractional amplitude of the periodic signal was increased until the periodogram showed a peak at that frequency above the previously determined $3 \sigma$ threshold over $98 \%$ of the time. For example, for the time sampling and duration corresponding to the observations on 2006 May 3, we find that we are able to detect a 20 minute periodic signal with an amplitude that is at least $20 \%$ of the maximum flux density (Figure 11). The sensitivity for the detection of a QPO increases with frequency because the underlying red noise component has less power at higher frequencies (e.g., a 10 (40) minute QPO signal will result in power greater than expected from red noise when its amplitude is 5\% (30\%) of the maximum flux density). Our sensitivity for the detection of a periodic signal is similar for the other observed light curves with similar durations ( $>2 \mathrm{hr}$ ).

We note that attempting to remove the broad, 40-100 minute emission peaks with a low-order functional fit to increase sensitivity to periodic signals will introduce a statistical bias, because that will only remove some combination of lowfrequency power without actually removing the entire red noise component. Because the light curves are consistent with a red noise process at all timescales, including the longer broad maxima, any statistical analysis must be performed without first modifying the light curves. For example, the light curve reported by Genzel et al. (2003), the first used to argue for the possible presence of a periodic variation, shows no significant periodicity when subjected to the red noise analysis described here. Figure 12 shows the analysis of this night using the light curve re-extracted by Meyer et al. (2006). A significant signal was found when a low-order polynomial fit to the light curve was removed in Meyer et al. (2006). However, this significance 

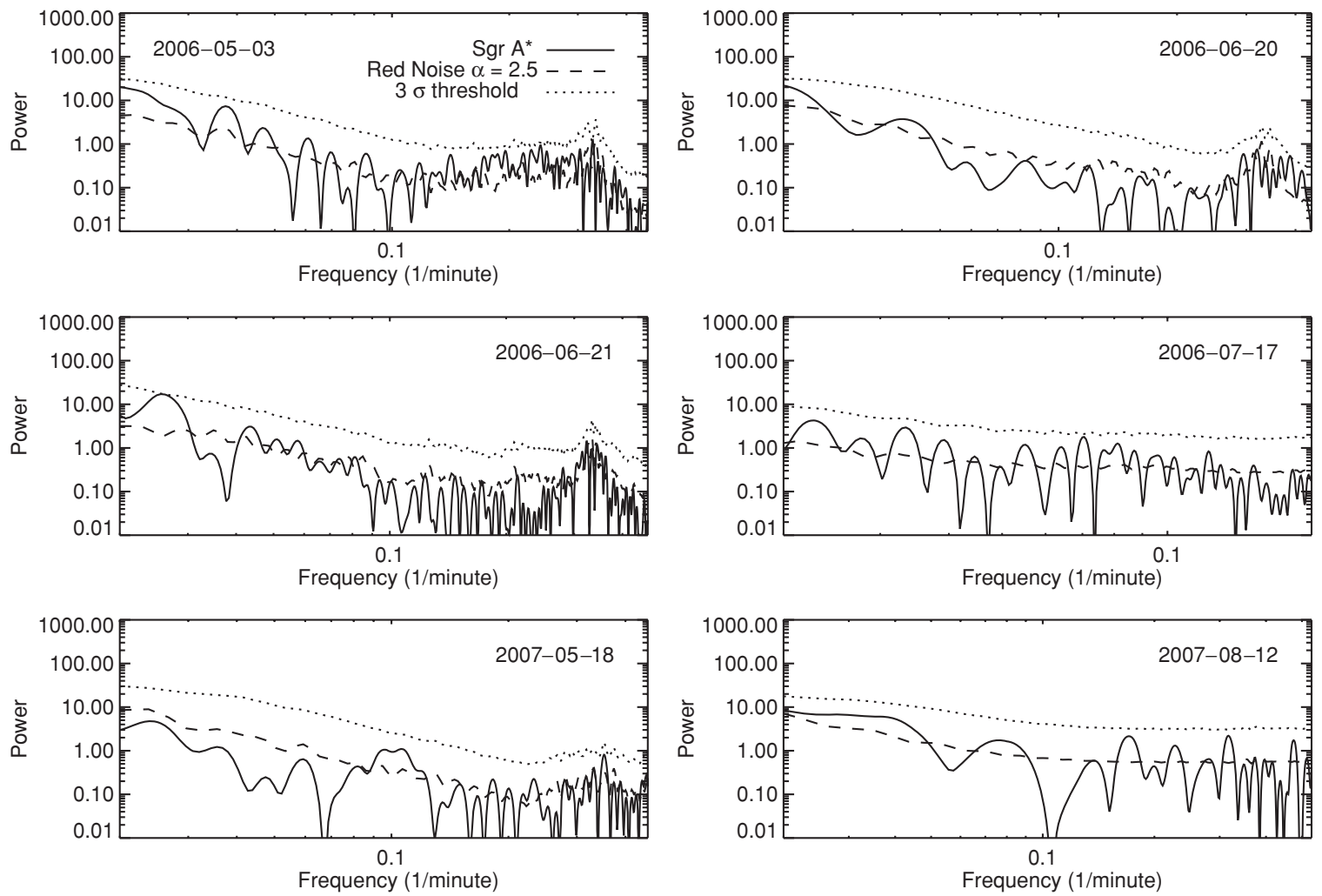

Figure 8. Normalized Lomb-Scargle periodograms of the Sgr A* light curves (black) at $K^{\prime}$. The $3 \sigma$ significance threshold determined from MC simulations of red noise with a power-law index, $\alpha=2.5$ is also plotted (dotted line). The average of $10^{5}$ periodograms with $\alpha=2.5$ at the same time sampling is also shown for comparison (dashed line).
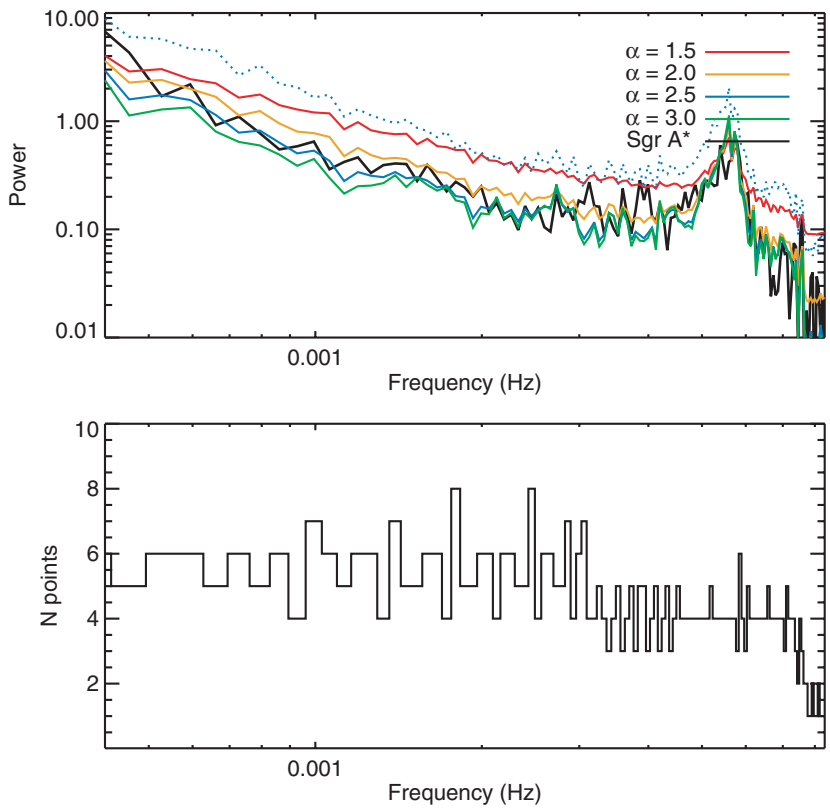

Figure 9. Top: Averaged periodogram from five nights of $K^{\prime}$ observations (black line) with durations greater than 80 minutes (resulting in the exclusion of 2007 August 12). The colored lines are the average of $10^{4} \mathrm{MC}$ simulations of combining data sets with the same sampling, for three different red noise power laws. The dotted blue line corresponds to the $3 \sigma$ threshold of power for red noise with $\alpha=2.5$. The large spike at $0.0056 \mathrm{~Hz}$ ( 3 minutes) is an artifact resulting from the regular dithers in the observations. Bottom: Plot of the number of points contributing to the averaged periodogram at each frequency.

may be biased by the removal of a number of low-frequency components from the light curve.

\subsubsection{Structure Function}

The first-order structure function is often used to determine the timescale and the intrinsic variability in AGN light curves (e.g., Simonetti et al. 1985; Hughes et al. 1992; Paltani 1999). Here, we use the structure function to determine the PSD slope for each night because it is more straightforward to calculate errors in the structure function. For a set of measurements, $s(t)$ at times $t$, the first-order structure function $V(\tau)$ is defined as

$$
V(\tau) \equiv\left\langle[s(t+\tau)-s(t)]^{2}\right\rangle .
$$

Because this data set is unevenly sampled, we determined the structure function by calculating $[s(t+\tau)-s(t)]^{2}$ for all possible pairs of time lags and place them into bins with widths of one fourth of the lag times. Variable bin sizes were chosen in order to more evenly distribute the number of points in each bin, since there are many more samples at small lags than at large lags. The median lag time in each bin is assigned to be the lag time for that bin, while the average of the $V(\tau)$ values in each bin is used as the value of the structure function at that lag. The error associated with each bin is $\sigma_{\text {bin }} / \sqrt{N_{\text {bin }}}$, where $\sigma_{\text {bin }}$ is the standard deviation of the $V(\tau)$ values in the bin and $N_{\text {bin }}$ is the number of points. We do not consider lags with fewer than five points in that bin. The resulting structure functions are presented in Figure 13.

Structure functions typically show a power-law portion and two plateaus - one at the timescale of the noise and one plateau at lags longer than the variability timescale of the underlying physical process. The power-law portion of the structure function is a measurement of the variability of the process. If the underlying process is stationary, then the logarithmic slope, $\beta$ (where $V(\tau) \propto \tau^{\beta}$ ), of this region can be related to the power 

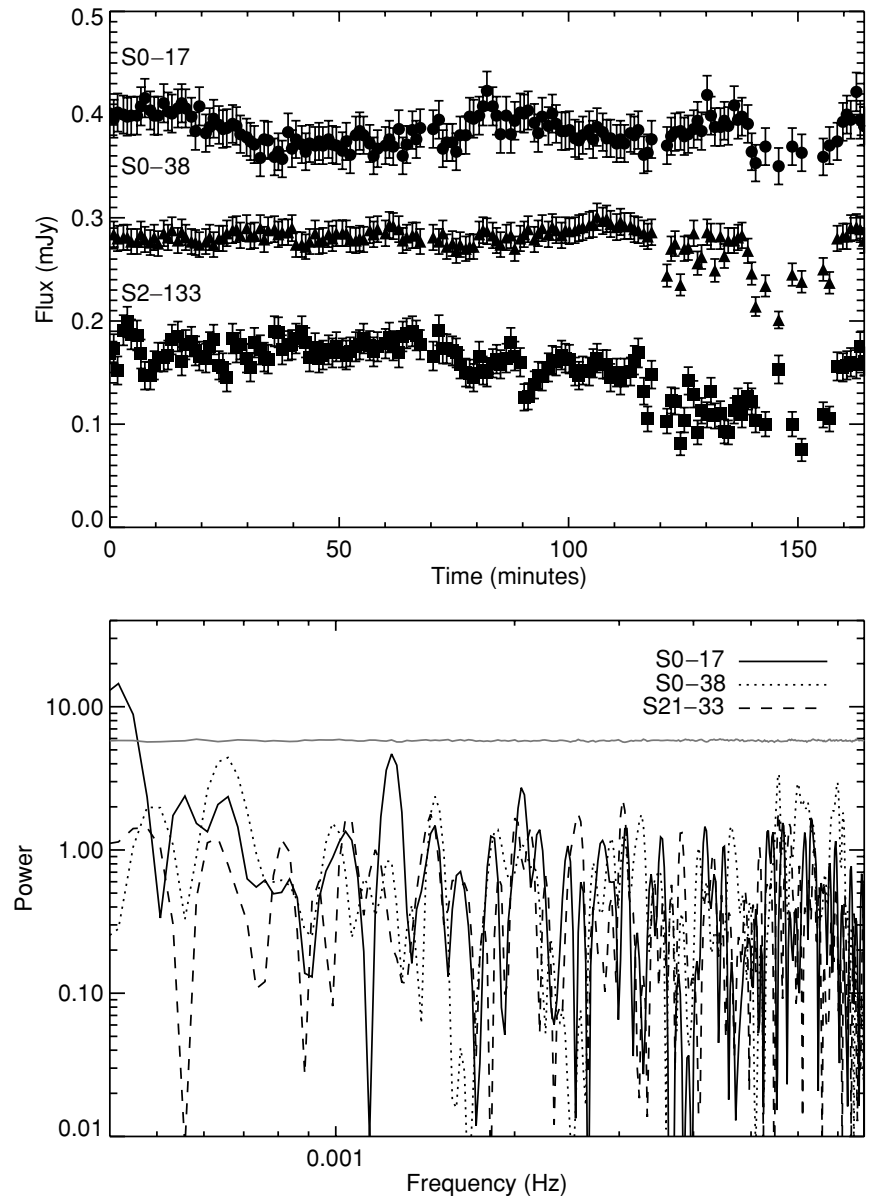

Figure 10. Top: Light curves of the stellar comparison sources from 2006 June 21. The light curve of S0-38 is offset by $0.18 \mathrm{mJy}$ for clarity. Bottom: Corresponding periodograms of the comparison sources. The $3 \sigma$ threshold for white noise $(\alpha=0)$ is in solid gray.

Table 2

PSD Fits from the Structure Function

\begin{tabular}{lccc}
\hline \hline $\begin{array}{l}\text { Date } \\
\text { (UT) }\end{array}$ & $\begin{array}{c}\text { Fit Range } \\
\text { (minutes) }\end{array}$ & $\beta^{\mathrm{a}}$ & $\alpha^{\mathrm{b}}$ \\
\hline 2006 May 3 & $1-20$ & $1.37 \pm 0.09$ & $2.74 \pm 0.13$ \\
2006 Jun 20 & $1-20$ & $1.40 \pm 0.08$ & $2.79 \pm 0.12$ \\
2006 Jun 21 & $1-15$ & $1.42 \pm 0.18$ & $2.79 \pm 0.27$ \\
2006 Jul 17 & $1-40$ & $0.71 \pm 0.09$ & $1.80 \pm 0.16$ \\
2007 May 18 & $1-20$ & $0.79 \pm 0.11$ & $1.95 \pm 0.20$ \\
2007 Aug 12 & $1-30$ & $0.26 \pm 0.04$ & $1.06 \pm 0.08$ \\
\hline
\end{tabular}

Notes.

${ }^{\text {a }}$ Slope of the power-law portion of the structure function, $V(\tau) \propto \tau^{\beta}$.

${ }^{\mathrm{b}}$ Slope of the PSD, $P(f) \propto f^{-\alpha}$.

index, $\alpha$ of the PSD $\left(P \propto f^{-\alpha}\right)$. Because the light curves in this study have finite sampling and duration, this relationship must be determined separately for each night. To convert the measured structure function slope into the PSD slope, we measured the slope of an average of $10^{3}$ structure functions $(\beta)$ from simulated light curves for a number of PSD slope values $(\alpha)$. The relationship between the measured structure function slope and the PSD slope is linear for values of $\alpha$ between 1.0 and 3.5 (Figure 14). These linear fits are used below to convert between the two slopes. We will use the structure function to compute the PSD because it is more straightforward than fitting the periodogram, given the limitations of the time sampling.
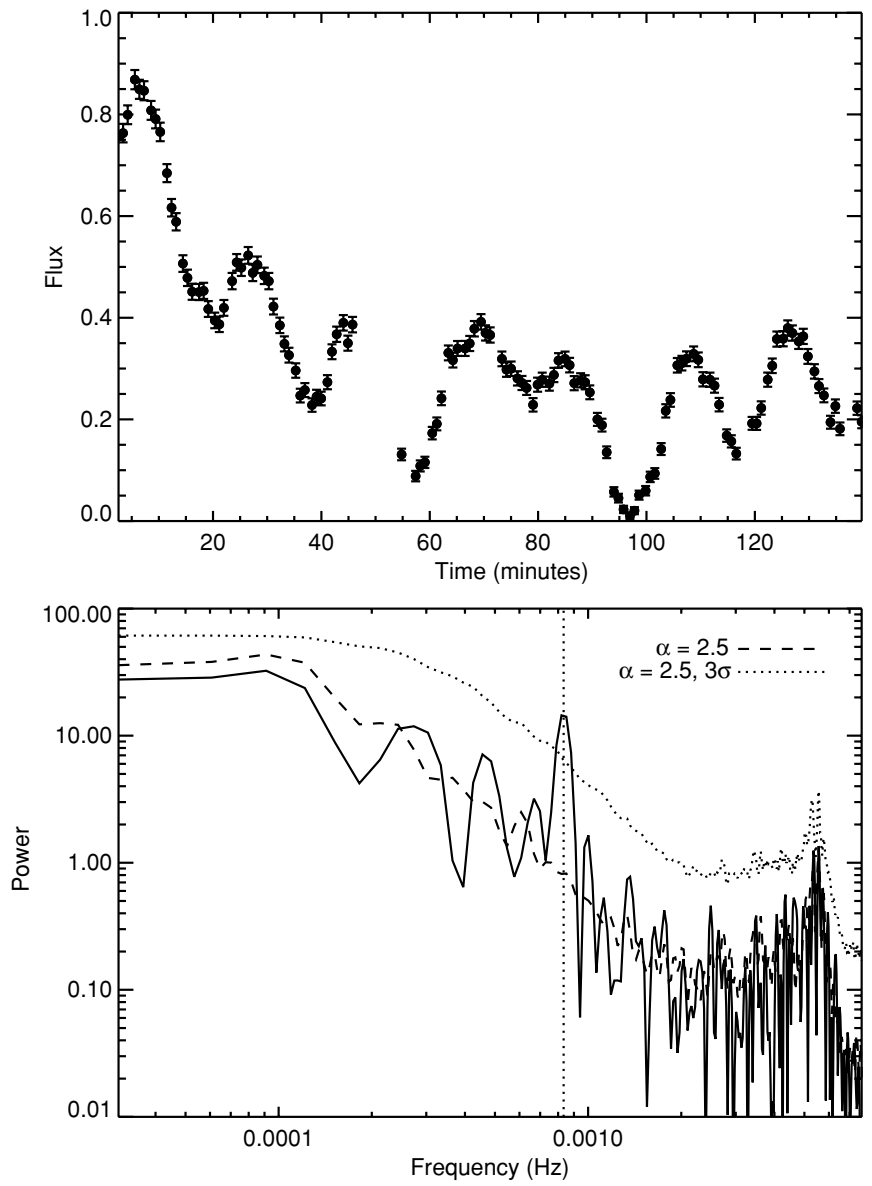

Figure 11. Top: Simulated light curve with the same time sampling as 2006 May 3 with a 20 minute periodic signal with an amplitude $15 \%$ of the maximum flux seen that night. Error bars in the light curve are based upon the noise properties of that night. Bottom: Corresponding periodogram of the simulated light curve (solid line) along with the average (dashed) and $3 \sigma$ threshold of red noise power from the MC simulations (dotted line). We can recover the signal at 20 minutes (vertical dotted line) at above our established confidence threshold.

The structure function for $\mathrm{Sgr} \mathrm{A}^{*}$ shows a power-law portion at periods greater than about 1 minute and a flattening at periods greater than about 40 minutes. This flattening is probably not a sign of a turnover in the intrinsic variability timescale, but is rather from the poor sampling at longer periods, as shown by large variations in the value of the structure function from MC simulations of red noise using the same time sampling. This suggests that $\operatorname{Sgr} \mathrm{A}^{*}$ is likely to be variable at all the timescales probed in this study. Fits were made to the powerlaw region of the structure function, typically up to about half or one-third of the total time of observations for each night, and are summarized in Table 2. The best-fit power-law index for the nights of 2006 May 3 and 2006 June 20, and 21 has $\beta=1.4 \pm$ $0.1(\alpha=2.7 \pm 0.1)$, between 1 and 20 minutes, consistent with the fit of $\alpha \approx 2.5$ for the slope of the combined periodogram. The other three nights have slopes ranging from $\beta \approx 0.8$ to $\beta \approx 0.3$. The night with the flattest slope (2007 August 12) is also the night where the emission from Sgr $\mathrm{A}^{*}$ was faint for the duration of the observation. Figure 15 shows that though the slope, $\beta=0.26 \pm 0.04$, is flatter than on other nights, it is significantly steeper than that of S0-17 $(\beta=0.04 \pm 0.04)$, S2-133 $(\beta=0.11 \pm 0.05)$, and simulations of white noise $(\beta=-0.01)$, which shows that the emission is not from a constant background source. The differences in slope between different nights may be attributable to the increase in photon 

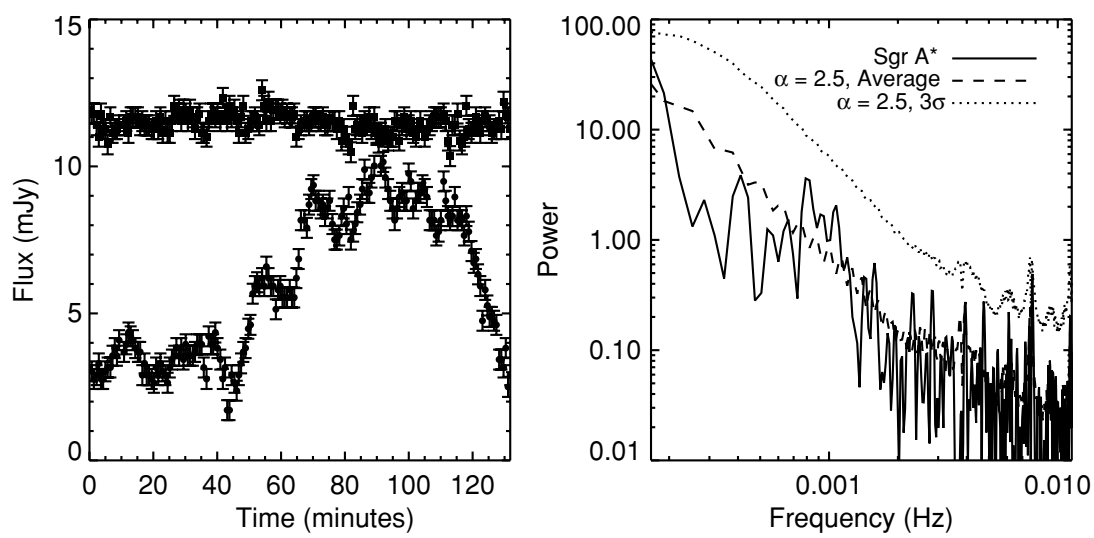

Figure 12. Left: Dereddened light curve of Sgr A*, from 2003 June 16, as extracted by Meyer et al. (2006; filled circles) along with a comparison source, called S1 in that paper (squares, offset by $6 \mathrm{mJy}$ for clarity). Right: Periodogram of the Sgr A* light curve (solid) with the mean power (dashed) and $3 \sigma$ thresholds set by MC simulations of red noise with a power-law slope of $\alpha=2.5$ (dotted).

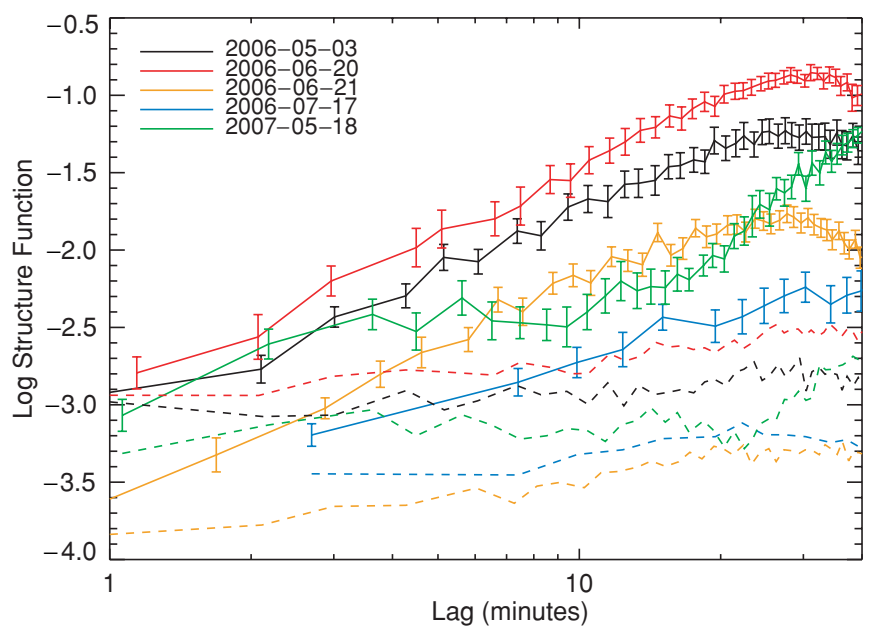

Figure 13. Structure functions for five of the $K^{\prime}$ nights for Sgr A* (solid) and the comparison source, S0-17 (dashed). Error bars are calculated from $\sigma / \sqrt{N}$, where $\sigma$ is the standard deviation and $N$ is the number of points in the bin. Error bars for S0-17 are of comparable sizes, but are omitted for clarity.

noise at these faint fluxes (see Section 4). For all nights, the S0-17 structure function is also flat, with a slight rise at longer timescales (also from poor sampling). The structure function of Sgr A* for the $L^{\prime}$ night on 2005 July 28 has a best-fit slope $\beta=0.77 \pm 0.18$, corresponding to $\alpha=1.84 \pm 0.27$, which is within the range of observed $K^{\prime}$ power-law slopes (Figure 16).

\subsubsection{Autocorrelation}

To compare the Sgr A* variability between each night, we computed the autocorrelation function for each observation. The autocorrelation is defined as

$$
\mathrm{AC}(\tau) \equiv\langle[s(t+\tau) s(t)]\rangle,
$$

where $s(t)$ is the measured flux at time, $t$, and $\tau$ is the time lag. Because the light curves are unevenly sampled, we computed the autocorrelation at all possible lags and then placed the pairs in bins, similar to our method for the computing of the structure function. We find that the correlation coefficients at timescales

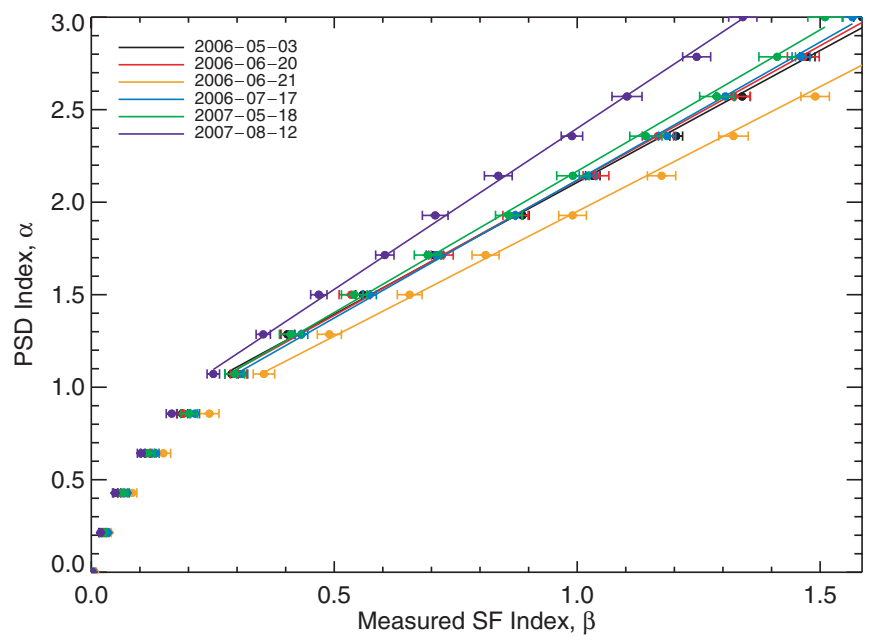

Figure 14. The relationship between intrinsic PSD power index and the measured power law of the structure function as simulated for the time sampling on each night. The line fits are made for PSD power-law slope $\alpha>1.0$ in the linear region of the relationship between the intrinsic PSD power law and the structure function power law.

up to 40 minutes are consistent across all nights. At time lags greater than 40 minutes, the autocorrelations appear to diverge from each other. This is mostly likely from poor sampling at those frequencies. From MC simulations of red noise with the same time sampling as the data set, we find that the lags greater than about 40 minutes have large variations in autocorrelations coefficients; we therefore defer interpretations of lags greater than 40 minutes until more data are available to better sample these timescales.

\section{DISCUSSION}

Our analysis shows that the Sgr A* NIR light curves observed in this study are entirely consistent with red noise with no significance evidence for a QPO signal near 20 minutes (or any other timescale). In addition, power is seen at the shortest timescales measured in the periodogram ( 2 minutes), well below the orbital period at the last stable circular orbit of a spinning black hole $(\sim 5-30$ minutes). This indicates that the highfrequency variability is not simply from Keplerian motion of a single-orbiting "hot spot" producing the NIR flux modulations (Meyer et al. 2006; Trippe et al. 2007). The high-frequency variability must originate from small areas of the accretion 


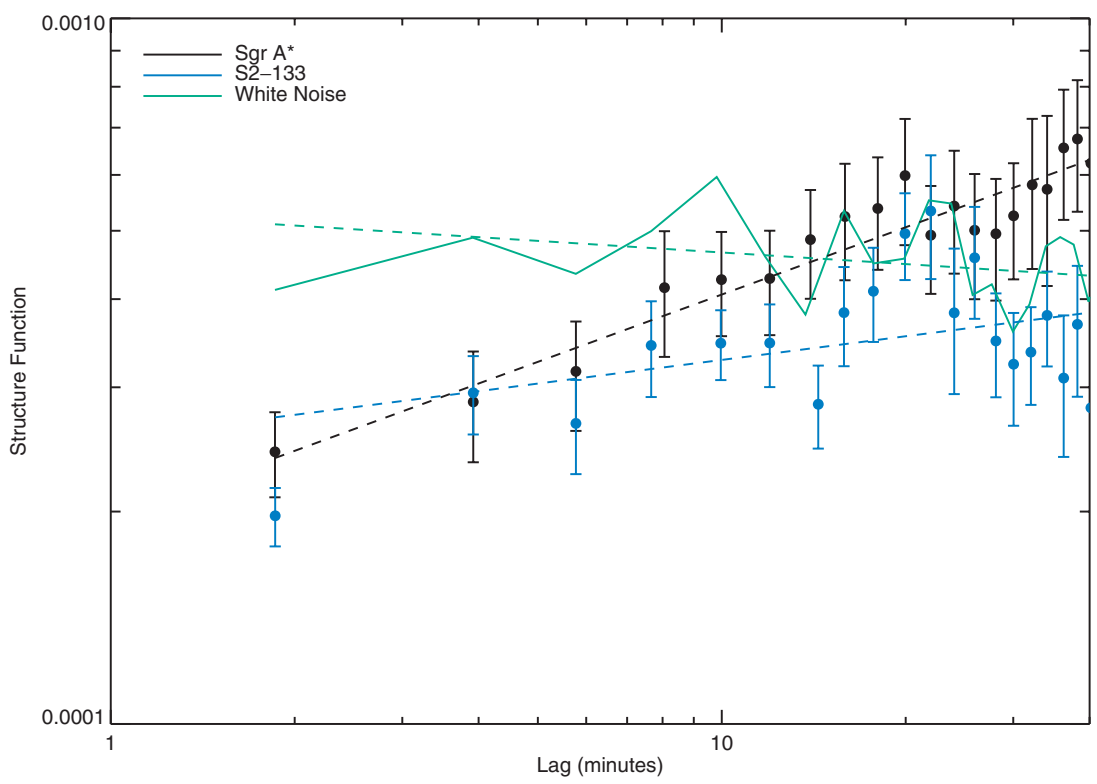

Figure 15. The $K^{\prime}$ structure function of Sgr A* (black), S2-133 (blue), and simulations of white noise (green) for 2007 August 12.

(A color version of this figure is available in the online journal.)

flow. In addition to power at high frequencies, the light curve for 2007 May 18 shows a strong spike in flux at about 10 minutes with a duration of $\sim 5$ minutes, and a rise time of only $\sim 3$ minutes, which also suggests that regions of the accretion flow responsible for the variability can be as small as $0.4 \mathrm{AU}$.

It is important to note that the significance threshold used in this study tests whether the periodogram power of peaks exceeds $3 \sigma$ at a given frequency. It does not take into account that we are scanning over a large range of frequencies. If one searches enough frequencies, a peak of arbitrary power will appear at a random frequency. If a peak in the periodogram was found in this study that may indicate a periodic signal, then additional simulations should be performed to determine its statistical significance by accounting for the range of frequencies probed. This is referred to as the false alarm probability in Horne \& Baliunas (1986) and has been calculated analytically for the case of white noise. In the case of red noise, the additional simulations would also need to account for the fact that larger peaks are more likely at low frequencies (Meyer et al. 2008).

The timing analysis of Sgr A* in the IR may be a way to compare the signatures of physical processes operating in the accretion flow of $\mathrm{Sgr} \mathrm{A}^{*}$ to those of AGNs. Because AGN variability studies are usually performed on X-ray observations, the ideal comparison of the variability of Sgr A* to that of AGNs should be done at X-ray energies. However, $\mathrm{X}$-ray emission from the inner accretion flow of Sgr A* can only be currently detected above the diffuse background during large flaring events. Simultaneous multiwavelength studies of Sgr A* have shown that every detectable X-ray flare shows corresponding peaks in the NIR, with no apparent time lag (Yusef-Zadeh et al. 2006; Eckart et al. 2006). The spectral slope is also similar at X-ray and IR wavelengths $\left(S_{v} \propto v^{-0.6}\right.$; Bélanger et al. 2005; Yusef-Zadeh et al. 2006; Hornstein et al. 2007), which supports a model in which the X-ray photons are the result of synchrotron self-Compton scattering of lower-energy photons from the population of electrons that produce synchrotron emission seen in the IR. If the IR and the X-ray emissions are produced by the same population of electrons, then our IR measurements can be used as a proxy for the X-ray variability; we find that the periodograms of the IR light curves of Sgr A* are in fact consistent with having intrinsic PSD slopes similar to those reported for AGNs at X-ray wavelengths $(\alpha \approx 1-3$; e.g., Lawrence \& Papadakis 1993; Kataoka et al. 2001; Markowitz et al. 2003). Our observations are consistent with Sgr A* being variable at all timescales between $\sim 2$ minutes and $\sim 1 \mathrm{hr}$ with no changes in PSD slope between these timescales during the observations period on any single night. This steeply rising power law cannot be extended to arbitrarily low frequencies because the integrated power would diverge. For example, AGNs typically show either one or two breaks in their power spectrum, with recent evidence suggesting that these breaks scale with the mass and accretion rate of the AGN (McHardy et al. 2006). The lack of a break in the Sgr A* periodogram is not unusual because the breaks seen in AGNs are at timescales on the order of days or weeks (Markowitz et al. 2003). Using the relationship between the break frequency, the black hole mass, and accretion rate found by McHardy et al. (2006) for stellar mass black holes and AGNs, the predict break frequency for Sgr A* is $\sim 1 \times 10^{6}$ days. However, a direct comparison to AGNs is difficult at this time because the physical scales involved are so different; the cores of AGNs in which variability is usually measured have sizes on the order of parsecs, whereas Sgr A* is unresolved in this study at a resolution of 65 mas, or $\sim 540 \mathrm{AU}$. The accretion luminosities of AGNs with well-measured timing properties are substantial fractions of their Eddington luminosity compared to the $10^{-9}$ Eddington luminosity for Sgr A*, which may make the comparison inapplicable as well. Nevertheless, the variability properties of $\mathrm{Sgr} \mathrm{A}^{*}$ are valuable additions to the broadband spectrum since any models that can produce the observed spectrum should also be able to account for the timing characteristics.

While it may appear from the results in Section 3.2.2 that the slope of the power spectrum is changing between nights, the red noise simulations show that, as the amplitude of the red noise signal decreases, photon noise will increasingly dominate the measurement of the slope, thus biasing the measurement of the true slope of the underlying red noise process. To determine when photon noise becomes important 


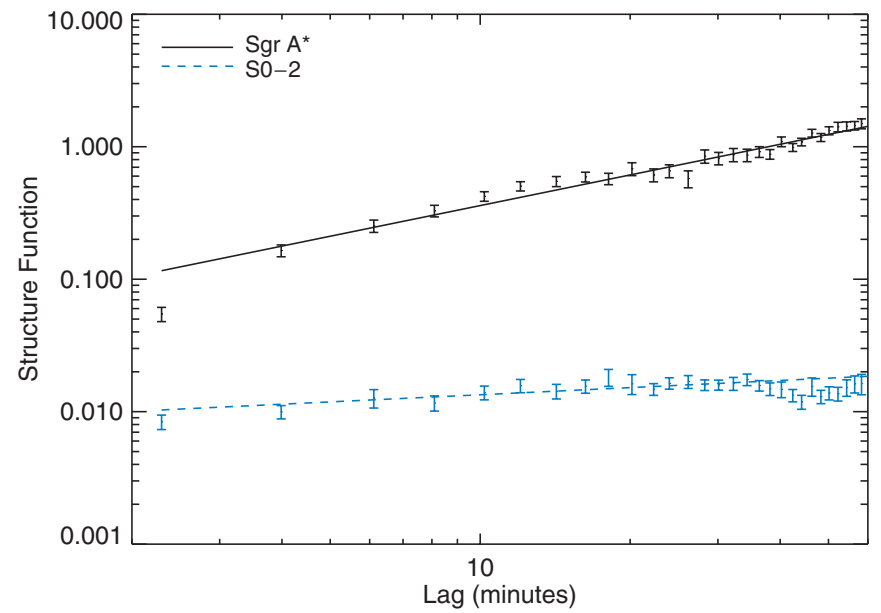

Figure 16. Structure function of Sgr A* (black), S0-2 (blue) at $L^{\prime}$ on 2005 July 28. The best-fit power law between 3 and 40 minutes is also plotted. The best-fit power law for $\operatorname{Sgr} \mathrm{A}^{*}$ is $\beta=0.77 \pm 0.18$, corresponding to $\alpha=1.84 \pm 0.27$ (see Section 3.2.2 for details).

(A color version of this figure is available in the online journal.)

to the slope measurements, we performed $\mathrm{MC}$ simulations of light curves with time sampling from 2008 August 12 and assuming an intrinsic $\alpha=2.5$ for various values of the ratio of the standard deviation of the red noise signal to the standard deviation of the expected measurement noise $\left(\sigma_{\text {red noise }} / \sigma_{\text {white noise }}\right)$. Average values of $\alpha$ were extracted from 200 simulated light curves at each value of $\sigma_{\text {red noise }} / \sigma_{\text {white noise }}$ from $0.1-100.0$ (Figure 17 ). We find that as $\sigma_{\text {red noise }} / \sigma_{\text {white noise }}$ becomes smaller, the measured value of $\alpha$ becomes flatter. The simulation showed that a measurement of $\alpha=1.0$ can arise even from a red noise process with $\alpha=2.5$ if $\sigma_{\text {red noise }} / \sigma_{\text {white noise }}=$ 1.1. We find that the measured value of $\alpha$ will asymptote to 2.5 when $\sigma_{\text {red noise }} / \sigma_{\text {white noise }}>7.9$. This result suggests that the measurement of $\alpha=1.06 \pm 0.08$ for 2007 August 12 can be explained as having the same intrinsic PSD slope, $\alpha$, as the other nights, but made flatter by a more prevalent white noise component. Note that this is an effect of a white noise source on top of a red noise signal, which can be caused simply by the measurement noise at faint flux densities or the presence of an unresolved stellar source. Figure 17 shows the relationship between the measured value of $\alpha$ and the ratio of standard deviation of the observed light curves to the expected noise level for the corresponding mean flux density $\left(\sigma_{\text {signal }} / \sigma_{\text {noise }}\right)$ for all $K^{\prime}$ nights. The measured value of $\alpha$ appears to asymptote on nights when the red noise amplitude is stronger. This relationship is consistent with that found in the MC simulations, where the nights with lower values of $\sigma_{\text {signal }} / \sigma_{\text {noise }}$ have flatter red noise spectra than those on the nights with greater fractional signal variance. These results show that the differences between the measured slopes for different nights may be explained by the effect of measurement noise on light curves with faint fluxes.

We also considered how a slightly time-correlated source would affect the power-law slope measurements by including a time-correlated source with a small $\alpha$ into the simulations and varying its signal strength as the above treatment with white noise. This is motivated by the fact that there is a slight slope to the structure function of our comparison source S2-133. For example on 2007 August 12, S2-133 has a structure function power-law slope of $\beta=0.11 \pm 0.05$ corresponding to $\alpha=0.7 \pm 0.2$. We find that the addition of such a background

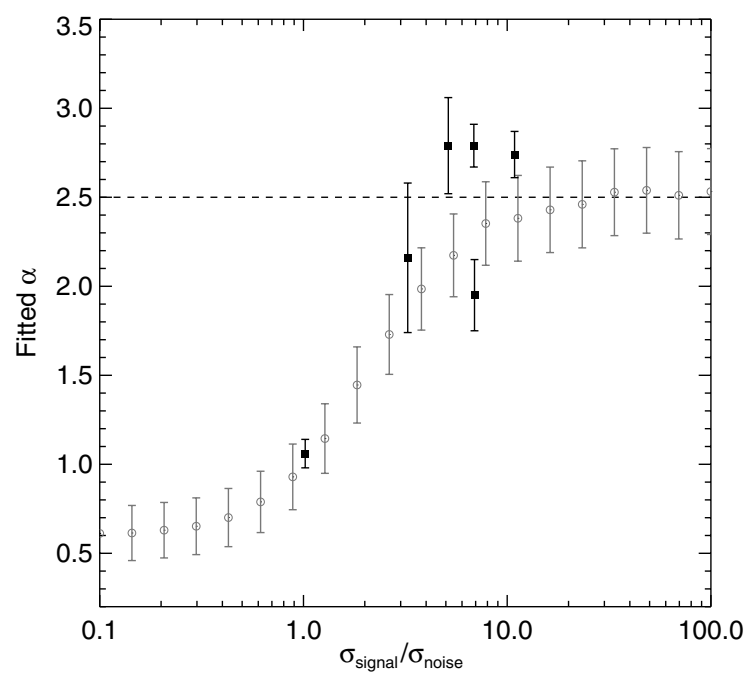

Figure 17. The plot of the fitted power-law slope $\alpha$ as a function of the ratio between the standard deviation of the light curve (signal) and the photon noise expected at the mean flux density for each $K^{\prime}$ night (solid black squares). The open gray circles show the results of MC simulations with the same time sampling as 2007 August 12, showing that as the red noise signal becomes comparable to the measurement noise, the measured slope of the power spectrum becomes flatter than the intrinsic value of $\alpha=2.5$ used in the simulation.

source increases the measured Sgr A* PSD power-law slope $\alpha$ by a maximum of 0.1 , which is about the same level as our fitting error. The effect of a slightly time-correlated background source thus appears to be a source of systematic error, but one which does not significantly affect our results.

A notable feature of the light curves in these observations is that there appears to be a lower limit, or "floor" to the light curves from each night. This lower limit is not an observational constraint, because we are able to reliably detect stars within $0^{\prime \prime} .5$ of $\mathrm{Sgr} \mathrm{A}^{*}$ to $K^{\prime} \approx 19 \mathrm{mag}$ compared to the faintest Sgr $\mathrm{A}^{*}$ observation at $K^{\prime} \approx 17 \mathrm{mag}$. A question to be investigated in future studies is whether this represents a "quiescent" state of Sgr A*, characterized by lower variability at that flux level or is a faint star confused with $\operatorname{Sgr} \mathrm{A}^{*}$. If the low flux density state is a physically distinct state, then the large flux variations would be the result of additional emission of energy above this quasisteady state. To examine the nature of this faint state, we can look at the peak of the distribution of flux densities fainter than $\sim 0.3 \mathrm{mJy}$ for each night. We find that the flux density of the peak of this distribution shifts slightly between different nights of observations. However, this shift appears only to be marginally significant given the lower photometric accuracy at the fainter flux levels. Before physical models can be applied to the faint state, a more detailed analysis of the light curves is necessary to establish whether this state exists. If the faint state of Sgr A* can be modeled as arising from the steady accretion of mass, then the slight drift of this mean flux level may be explained by a gradual change in the accretion rate. Another explanation for the drift in the mean Sgr A* flux density is that it is due to very long timescale flux variation from the red noise power-law behavior of the flux as in AGNs.

It is clear that $\mathrm{Sgr} \mathrm{A}^{*}$ can now always be detected in the IR using LGS AO and that even at low flux densities, the $K^{\prime}-L^{\prime}$ color of the source appears to be redder and have PSD slopes steeper than a star, but the measurement errors do not rule out the possibility of a faint stellar component in the NIR flux from the location of Sgr A*. More observations during both the faint and bright states of Sgr A* are necessary to 
confirm whether there truly exists a quiescent state with different timing behavior. Since Sgr A* is much redder than any stellar source, observations at $L^{\prime}$ would be much less affected by stellar contamination than at $K^{\prime}$, thus the test for a quiescent state may be more conclusive at $L^{\prime}$. Note that the existence of a quiescent state does not affect the timing analysis for periodic signals because the mean flux density is subtracted from a light curve to remove the zero frequency power before creating the periodogram.

While no QPO signal was detected in this study, this does not preclude the possibility for a transient periodic signal in other observations. In addition, a periodic signal with an amplitude below our detection threshold would also be difficult to detect unless the signal is consistently at the same frequency such that it would become statistically significant in a combined periodogram. More observations of Sgr A* would be helpful to investigate such low-amplitude QPO signals.

\section{CONCLUSION}

We have obtained seven NIR light curves of Sgr A* using NGS and LGS AO and through MC simulations, test for a periodic signal in the presence of red noise with unevenly sampled data. We find no statistically significant QPO signals in any of the Sgr A* light curves observed in this study; the variability appears to be consistent with a physical process having a power spectrum index $\alpha \sim 2.5$ in the averaged periodogram, with variations between $\alpha=1.0$ and 2.8 between different nights. A determination of the true PSD power-law slope of Sgr A* will require at least double the number of light curves observed in this study so that more sophisticated spectral estimation techniques can be used (e.g., Priestley 1981; Papadakis \& Lawrence 1993). While more data will be necessary to reliably determine the intrinsic PSD slope, it is unnecessary to invoke a periodic signal to describe the variability of the light curves observed in this study. We have also shown the importance of performing statistical tests for QPO signals using the entire Sgr A* light curve. Removing broad largeamplitude peaks eliminates some combination of low-frequency power, artificially boosting the significance of mid-range frequencies without actually removing the underlying red noise component.

The mechanism producing the IR variability in $\mathrm{Sgr} A *$ in these observations may be related to the processes occurring in AGNs or accreting black hole binaries, based on the similarity of their timing properties. Detailed model comparison between Sgr A* and other accreting black hole systems will require continuous observations over longer timescales and at faster time sampling to detect possible low- and high-frequency breaks, respectively, in the power spectrum.

The authors thank the staff of the Keck observatory, especially Randy Campbell, David LeMignant, Jim Lyke, Steven McGee, and Hien Tran for all their help in obtaining the new observations and Guillaume Bélanger and Matt Malkan for helpful discussions. Support for this work was provided by NSF grant AST-0406816 and the NSF Science \& Technology Center for AO, managed by UCSC (AST-9876783), and the LevineLeichtman Family Foundation. The IR data presented herein were obtained at the W. M. Keck Observatory, which is operated as a scientific partnership among the California Institute of Technology, the University of California and the National Aeronautics and Space Administration. The Observatory was made possible by the generous financial support of the W. M.
Keck Foundation. The authors recognize and acknowledge the very significant cultural role that the summit of Mauna Kea has always had within the indigenous Hawaiian community. We are most fortunate to have the opportunity to conduct observations from this mountain.

Facilities: Keck:II (NIRC2).

\section{REFERENCES}

Armitage, P. J., \& Reynolds, C. S. 2003, MNRAS, 341, 1041

Aschenbach, B., Grosso, N., Porquet, D., \& Predehl, P. 2004, A\&A, 417, 71

Bélanger, G., Goldwurm, A., Melia, F., Ferrando, P., Grosso, N., Porquet, D., Warwick, R., \& Yusef-Zadeh, F. 2005, ApJ, 635, 1095

Bélanger, G., et al. 2009, ApJ, submitted

Benlloch, S., Wilms, J., Edelson, R., Yaqoob, T., \& Staubert, R. 2001, ApJ, 562, L121

Baganoff, F. K., et al. 2003, ApJ, 591, 891

Blum, R. D., Sellgren, K., \& Depoy, D. L. 1996, ApJ, 470, 864

Diolaiti, E., Bendinelli, O., Bonaccini, D., Close, L., Currie, D., \& Parmeggiani, G. 2000, A\&AS, 147, 335

Eckart, A., Schödel, R., Meyer, L., Trippe, S., Ott, T., \& Genzel, R. 2006, A\&A, 455, 1

Eisenhauer, F., et al. 2005, ApJ, 628, 246

Eckart, A., et al. 2006, A\&A, 450, 535

Eckart, A., et al. 2008, A\&A, 479, 625

Falanga, M., Melia, F., Tagger, M., Goldwurm, A., \& Bélanger, G. 2007, ApJ, 662, L15

Genzel, R., Schödel, R., Ott, T., Eckart, A., Alexander, T., Lacombe, F., Rouan, D., \& Aschenbach, B. 2003, Nature, 425, 934

Ghez, A. M., Salim, S., Hornstein, S. D., Tanner, A., Lu, J. R., Morris, M., Becklin, E. E., \& Duchêne, G. 2005a, ApJ, 620, 744

Ghez, A. M., et al. 2003, ApJ, 586, L127

Ghez, A. M., et al. 2005b, ApJ, 635, 1087

Ghez, A. M., et al. 2008, ApJ, 689, 1044

Horne, J. H., \& Baliunas, S. L. 1986, ApJ, 302, 757

Hornstein, S. D., Ghez, A. M., Tanner, A., Morris, M., Becklin, E. E., \& Wizinowich, P. 2002, ApJ, 577, L9

Hornstein, S. D., Matthews, K., Ghez, A. M., Lu, J. R., Morris, M., Becklin, E. E., Rafelski, M., \& Baganoff, F. K. 2007, ApJ, 667, 900

Hughes, P. A., Aller, H. D., \& Aller, M. F. 1992, ApJ, 396, 469

Kataoka, J., et al. 2001, ApJ, 560, 659

Lawrence, A., \& Papadakis, I. 1993, ApJ, 414, L85

Lyubarskii, Y. E. 1997, MNRAS, 292, 679

Markowitz, A., et al. 2003, ApJ, 593, 96

Marrone, D. P., et al. 2008, ApJ, 682, 373

Mauerhan, J. C., Morris, M., Walter, F., \& Baganoff, F. K. 2005, ApJ, 623, L25

McHardy, I. M., Koerding, E., Knigge, C., Uttley, P., \& Fender, R. P. 2006, Nature, 444, 730

Melia, F., \& Falcke, H. 2001, ARA\&A, 39, 309

Meyer, L., Do, T., Ghez, A., Mark, M. R., Witzel, G., Eckart, A., Bélanger, G., \& Schödel, R. 2008, ApJ, 688, L17

Meyer, L., Eckart, A., Schödel, R., Duschl, W. J., Mužić, K., Dovčiak, M., \& Karas, V. 2006, A\&A, 460, 15

Moneti, A., Stolovy, S., Blommaert, J. A. D. L., Figer, D. F., \& Najarro, F. 2001, A\&A, 366, 106

Paltani, S. 1999, BL Lac Phenom., 159, 293

Papadakis, I. E., \& Lawrence, A. 1993, MNRAS, 261, 612

Press, W. H. 1978, Comm. Astrophys., 7, 103

Press, W. H., \& Rybicki, G. B. 1989, ApJ, 338, 277

Priestley, M. B. 1981, Spectral Analysis and Time Series, Vol. 1 \& 2, (London: Elsevier Academic Press)

Rafelski, M., Ghez, A. M., Hornstein, S. D., Lu, J. R., \& Morris, M. 2007, ApJ, 659,1241

Rieke, G. H., \& Lebofsky, M. J. 1985, ApJ, 288, 618

Schödel, R., et al. 2002, Nature, 419, 694

Schödel, R., Ott, T., Genzel, R., Eckart, A., Mouawad, N., \& Alexander, T. 2003, ApJ, 596, 1015

Simonetti, J. H., Cordes, J. M., \& Heeschen, D. S. 1985, ApJ, 296, 46

Timmer, J., \& Koenig, M. 1995, A\&A, 300, 707

Trippe, S., Paumard, T., Ott, T., Gillessen, S., Eisenhauer, F., Martins, F., \& Genzel, R. 2007, MNRAS, 375, 764

Uttley, P., McHardy, I. M., \& Papadakis, I. E. 2002, MNRAS, 332, 231

van Dam, M. A., et al. 2006, PASP, 118, 310

Vaughan, S. 2005, A\&A, 431, 391

Vaughan, S., Fabian, A. C., \& Nandra, K. 2003, MNRAS, 339, 1237

Yusef-Zadeh, F., et al. 2006, ApJ, 644, 198

Wizinowich, P. L., et al. 2006, PASP, 118, 297 